\title{
Les ancêtres du Christ. Chrétiens et Juifs dans la Chapelle Sixtine
}

Giovanni Careri

\section{(2) OpenEdition}

1 Journals

Édition électronique

URL : http://journals.openedition.org/imagesrevues/1601

DOI : 10.4000/imagesrevues. 1601

ISSN : 1778-3801

Éditeur:

Centre d'Histoire et Théorie des Arts, Groupe d'Anthropologie Historique de l'Occident Médiéval, Laboratoire d'Anthropologie Sociale, UMR 8210 Anthropologie et Histoire des Mondes Antiques

\section{Référence électronique}

Giovanni Careri, «Les ancêtres du Christ. Chrétiens et Juifs dans la Chapelle Sixtine », Images Re-vues [En ligne], 9 | 2011, mis en ligne le 05 août 2014, consulté le 30 janvier 2021. URL : http:// journals.openedition.org/imagesrevues/1601 ; DOI : https://doi.org/10.4000/imagesrevues.1601

Ce document a été généré automatiquement le 30 janvier 2021.

Images Re-vues est mise à disposition selon les termes de la Licence Creative Commons Attribution Pas d'Utilisation Commerciale 4.0 International. 


\title{
Les ancêtres du Christ. Chrétiens et Juifs dans la Chapelle Sixtine ${ }^{1}$
}

\author{
Giovanni Careri
}

Parmi les milliers de personnages peints dans la Chapelle Sixtine, ceux qui sont représentés dans les lunettes et les cintres de la voûte semblent être sans rapport ni de temps ni de lieu avec le contexte héroïque de l'ensemble. On y voit des familles prises dans leur vie intime, des femmes s'occupant de leurs enfants ou de

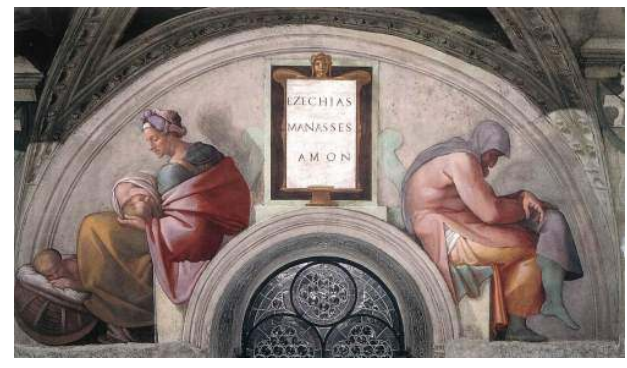
travaux domestiques, des vieillards mélancoliques endormis, des personnages errants, des hommes et des femmes dans l'attente (fig.1). 
Fig. 1

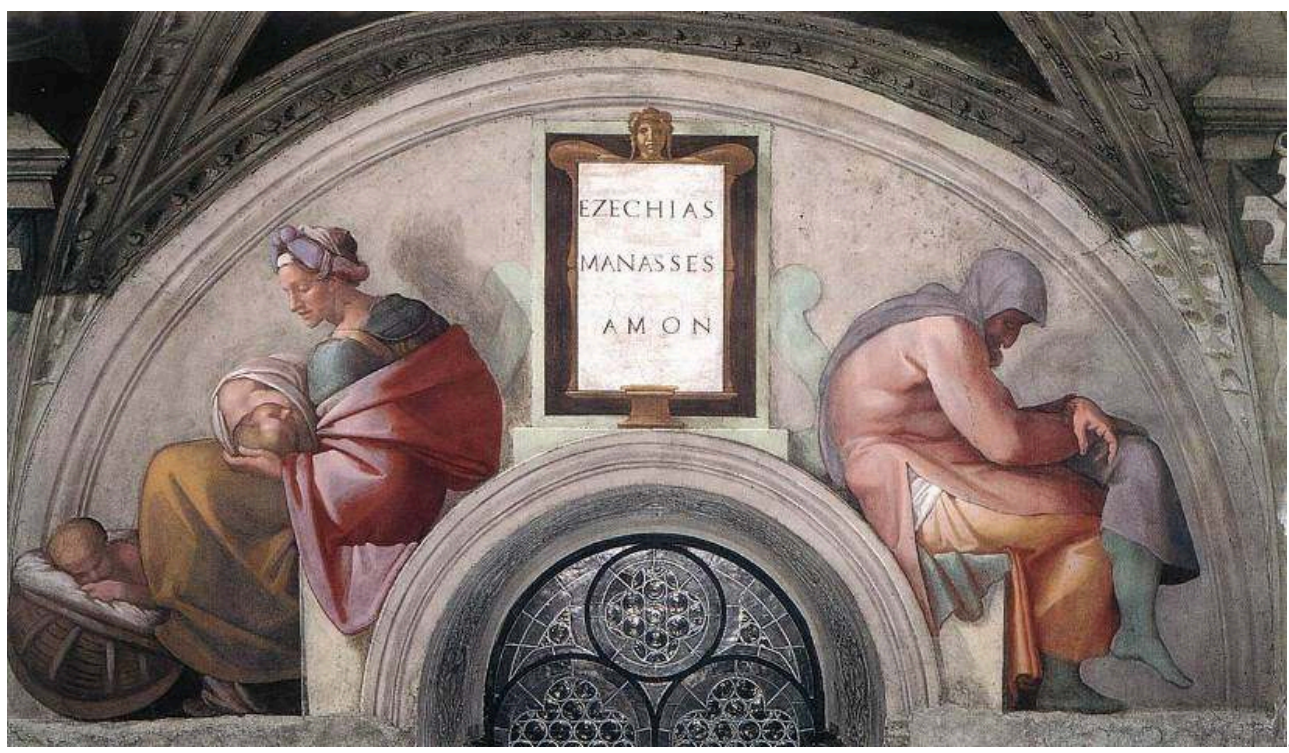

Michel-Ange, Ancêtres du Christ, 1508-12, Chapelle Sixtine, Rome

2 Nombre d'entre eux ont les bras et les jambes croisés, comme pour témoigner d'un empêchement à l'action. Leurs figures accablées projettent sur les murs des ombres denses, autant de marques d'une corporéité terrestre et d'une «immersion» dans le temps de la vie de tous les jours. Certaines de ces figures ont des traits sémites stéréotypés, marqués au point de rappeler des représentations anti-hébraïques de l'époque. La difficulté à comprendre les attitudes de ces figures s'exprime depuis le commentaire contenu dans la deuxième édition des Vies de Giorgio Vasari (1568) où elles sont qualifiées de " caprices extraordinaires et nouveaux ", c'est-à-dire, comme le souligne Paola Barocchi, selon la catégorie de l'invention formelle et iconographique par rapport à des modèles préexistants ${ }^{2}$. A l'époque romantique, après une longue période de silence, les figures des Ancêtres du Christ furent considérées comme des scènes de "réalisme domestique ${ }^{3}$, mais il faudra attendre l'affaire Dreyfus pour qu'Emile Zola fasse apparaitre l'aspect juif du cycle dans un passage de Rome, publié en 1896.

D'ailleurs, dans les cintres des fenêtres, dans les lunettes, des figures de beauté, de puissance et de grâce, naissaient encore, se pressaient, abondaient, les ancêtres du Christ, les mères songeuses aux beaux enfants nus, les hommes aux regards lointains, fixés sur l'avenir, la race punie, lasse, désireuse du Sauveur promis; tandis que, dans les pendentifs des quatre angles, s'évoquaient, vivantes, des scènes bibliques, les victoires d'Israël sur l'esprit du mal. ${ }^{4}$

En 1903, Charles Holroyd y voyait la "vie de l'homme sur terre» et allait jusqu'à imaginer l'artiste employé à transposer, dans les lunettes et les cintres, des scènes de marché recueillies et esquissées sur le vif dans le quartier du Borgo ou près du Castel sant'Angelo ${ }^{5}$. Pendant la seconde guerre mondiale cette vision a été corrigée au nom d'une analyse plus raffinée de la construction formelle et chromatique des figures, même si en 1942, Aldo Bertini parle encore d'un rapport à la vie quotidienne notamment dans les cintres : «[dans les lunettes] apparaît ainsi une humanité fatiguée et douloureuse, moins épique que celle de la voûte, moins mélancolique que celle des cintres, dans laquelle des motifs inspirés de la vie de tous les jours sont élevés au rang de composition d'une extrême beauté $»^{6}$ (fig. 2). 
Fig. 2

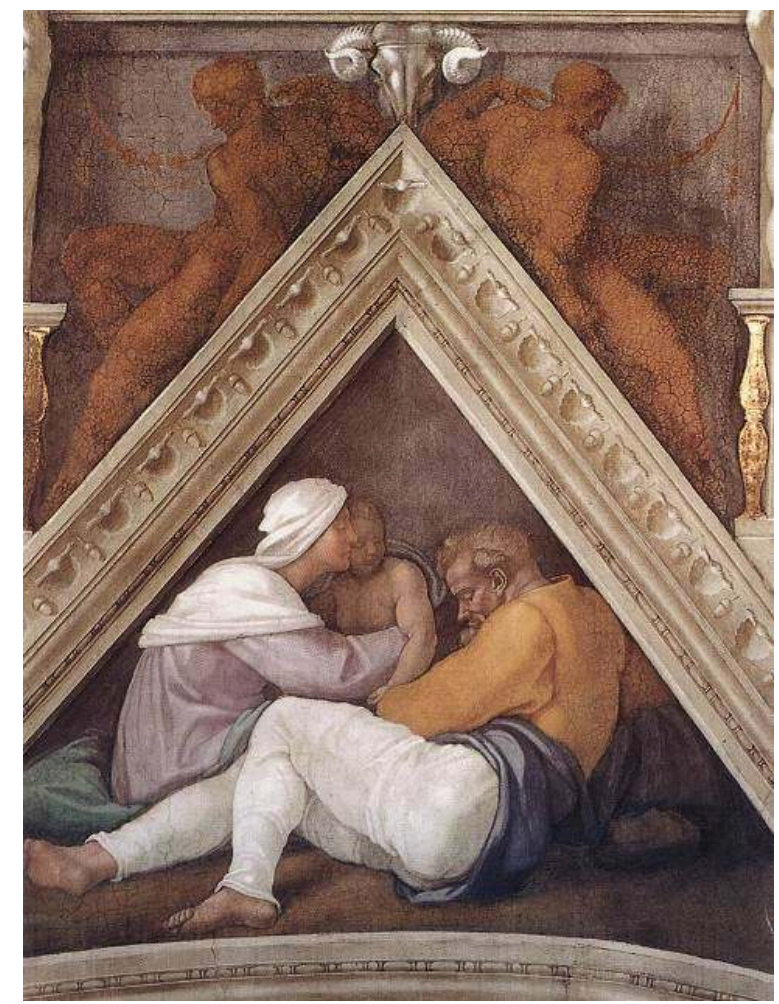

Michel-Ange, Ancêtres du Christ, 1508-12, Chapelle Sixtine, Rome

En 1945, Charles de Tolnay a soutenu qu'il fallait parler de création de types de la vie quotidienne plutôt que d'esquisses prises sur le vif. Conformément à son analyse néoplatonicienne de la chapelle, Tolnay exclut les lunettes et les cintres du monde idéal de la partie supérieure de la voûte. Un extrait de sa description mérite d'être cité parce qu'il contient, à mon avis, un écho des événements tragiques de la seconde guerre mondiale et de la persécution des juifs :

Dans les espaces réduits des cintres, Michel-Ange représente la vie domestique d'une humanité nomade. Tous semblent accablés par une grande fatigue; leurs âmes sont en proie à l'angoisse éternelle de ceux qui sont condamnés à l'errance comme s'ils étaient persécutés. [...] Nous sommes face au monde du devenir éternel, un monde fugace, sans souvenirs, sans histoire, sans espoir et sans but ${ }^{7}$.

Après ce texte, la dimension "domestique " et la dimension "judaïque » ont été complètement écartées, faisant place à une diatribe passionnante entre iconologues. En effet depuis 1944, au nom d'une méthodologie philologique et iconologique rigoureuse, Edward Edgar Wind avait rompu tout lien avec «la vie concrète » en proposant une interprétation allégorique des figures à partir des traductions latines des quarante noms juifs des Ancêtres du Christ inscrits dans les cartouches des lunettes. Cette liste, réduite à trente-trois après la destruction de deux lunettes pour faire place au Jugement dernier, est conforme à la généalogie qui descend d'Abraham à David et de David à Joseph et qui ouvre l'évangile de Mathieu. Selon Edgar Wind la figure peinte dans la lunette qui porte le nom d'Aminadab serait une allégorie de "l'attachement aux biens terrestres » (fig. 3). 
Fig. 3

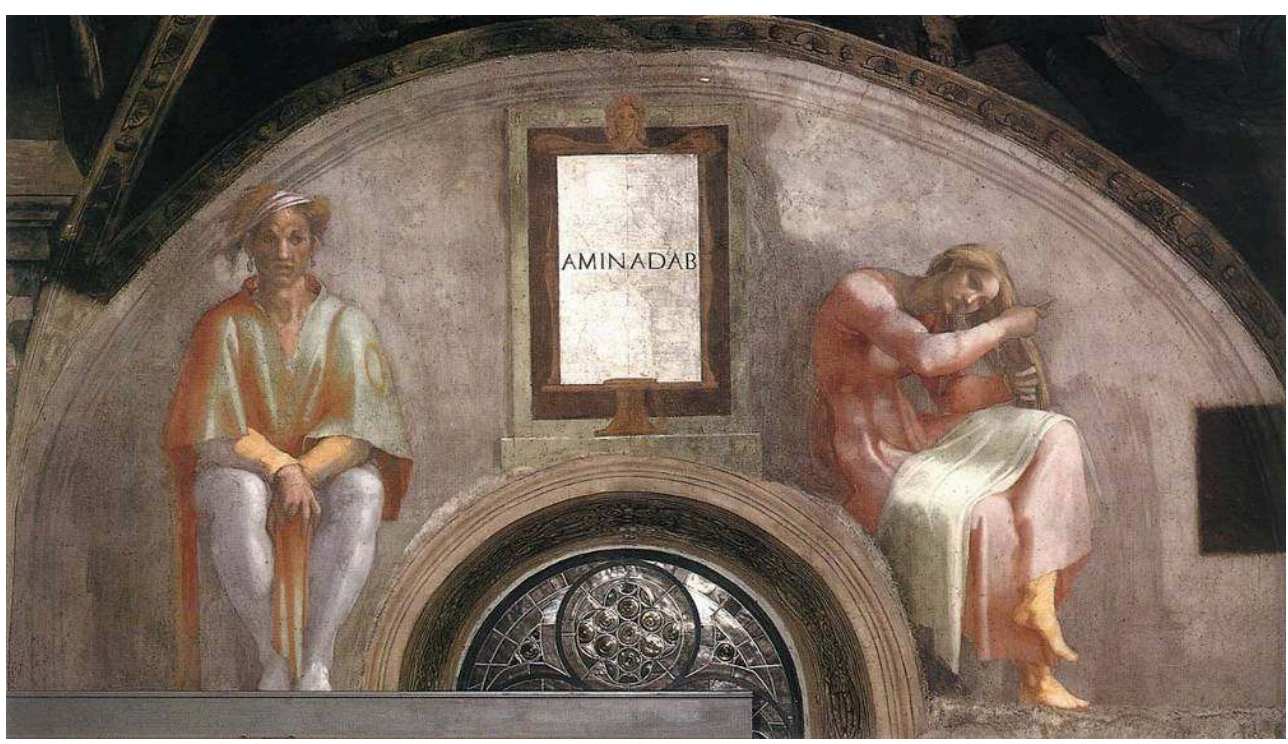

Michel-Ange, Ancêtres du Christ, 1508-12, Chapelle Sixtine, Rome

Dans le projet de Michel-Ange, à chaque image d'un vice s'oppose une image de vertu, les deux étant nommés par des noms juifs. Le nom d'Aminadab, par lequel commence la série, est associé à l'image d'une jeune femme avec un linge sur les genoux en train de se coiffer, tandis qu'un homme, assis immobile à côté d'elle, regarde devant lui sans rien faire. Aminadab est traduit par populus meus (mon peuple) et ces mots se trouvent dans la bible, dans le livre de Jérémie : "une vierge oublie-t-elle ses ornements, une épouse sa ceinture ? Et pourtant mon peuple m'a oublié pendant d'interminables jours" (Jérémie 2, 32). Le couple représente donc les vices de l'attachement aux biens ${ }^{8}$.

En appliquant la même méthode à d'autres textes bibliques, Frederick Hartt parvient cependant à des conclusions opposées : Aminadab ne serait pas la représentation d'un vice mais une préfiguration du Christ :

L'ancêtre suivant est le jeune Aminadab, assis droit, le regard fixé dans le vide pendant que sa femme peigne ses longs cheveux blonds. Aminadab signifie: "mon peuple est animé par une forte volonté (soit spontanée, soit déterminée du dehors)". Ce terme renvoie à la spontanéité du sacrifice du Christ. [...] La seule autre référence à Aminadab se trouve dans le Cantique des cantiques: "je suis confuse, mon désir m'a placée sur les 'chariots' d'Aminadab”. Selon Grégoire le Grand, ces chariots sont les quatre évangiles et la Synagogue est troublée car elle ignorait le sacrifice du Christ. L'interprétation de Grégoire le Grand explique la posture et le regard d'Aminadab, profondément impressionné par la révélation dont il avait ignoré le sens. Mais qui est la belle femme qui regarde devant elle pendant qu'elle se coiffe? Dans le Cantique des cantiques, un peu avant la référence à Aminadab, on peut lire: "qui est celle qui pointe comme l'aurore, belle comme la lune, éclatante comme le soleil, terrible comme troupes et étendards déployés?". Non seulement ce passage a été interprété comme une prophétie de la Vierge (et de l'église), mais ce texte était lu pendant la liturgie de l'Assomption à laquelle la chapelle était dédiée. On verra à travers toute la série des ancêtres que les hommes préfigurent le Christ et les femmes Marie ${ }^{9}$.

7 Le personnage représenté auprès de l'inscription du nom Aminadab est-il donc une représentation allégorique de l'oubli du divin ou bien une figuration typologique du Christ $^{10}$ ? La divergence entre ces interprétations de Edgar Wind et de Frederick Hartt a 
débouché sur une polémique violente, portant aussi sur l'identité du savant qui aurait donné à Michel-Ange la traduction en latin des noms hébreux.

En refusant implicitement la réduction du travail figuratif à la simple illustration des noms, Sidney J. Freedberg a proposé en 1961, une brève considération à propos de la « domesticité » des figures des Ancêtres qu'il définit comme « beings as unexceptionnal as possible " pour un artiste tel que Michel-Ange. La composante sémite serait, selon lui, une façon d'accentuer la " common humanity » de ces figures qui exprimeraient la lassitude et la crise créative de Michel-Ange lui même ${ }^{11}$. Dans les paroles de Zola sur la «race punie, lasse, désireuse du Sauveur promis» ou dans celles de Tolnay qui en rappelle les " pérégrinations continues » semblent cependant affleurer les traces d'une opération idéologique selon laquelle, comme le suggère Sidney Freedberg, la construction « extraordinaire et nouvelle » d'une « common humanity " passe à travers l'invention de figures qui sont de "simples figures domestiques" précisément parce qu'elles sont dotées de «semitic caracters». Mais avant qu'on ait pu tenter de construire le lien entre généalogie, domesticité et judaïsme il a fallu sortir de la perspective de la dénomination ${ }^{12}$. Dans un texte de 1994, Creighton Gilbert a été le premier à proposer de suspendre l'attribution d'un nom à chaque figure en suggérant de voir les Ancêtres comme un chœur, lié pour des raisons d'exégèse numérologique aux stations du peuple juif en exil dans le livre des Nombres. Dans cet essai l'auteur critique la tentative d'Esther Gordon Dotson de partager les figures en deux groupes: les familles représentées dans les cintres se dirigeant vers la Cité de Dieu et celles des lunettes orientées vers la Cité terrestre. Bien qu'effectivement elle soit trop étroitement dépendante du texte augustinien, l'interprétation de Dotson est pourtant très intéressante dans la mesure où elle nous invite à situer les Ancêtres dans le cadre plus général du dessein historique de la Chapelle Sixtine ${ }^{13}$. Les travaux de Dotson et de Gilbert ont enfin permis de sortir les Ancêtres du déterminisme nominal qui les avait réduits au rang d'illustrations de sources écrites plus ou moins rares.

Quant à la dimension «domestique » du cycle pour laquelle il n'existe pas de sources textuelles directes, on peut dire qu'elle n'a jamais vraiment été abordée. Je tenterai d'en rendre compte à travers une recherche moins subordonnée aux sources écrites et plus sensible au «travail » des images elles-mêmes. La même démarche me conduira à saisir les implications de la composante anti-hébraïque laquelle, par sa complexité intrinsèque, pose un défi à la méthodologie standard qui a souvent tendance à réduire la stratification de multiples schémas iconographiques à une seule signification.

Malgré une meilleure visibilité, rendue possible par la restauration commencée en 1980, une analyse rapprochée de ce cycle et des dispositifs visuels qui s'y manifestent ne semble pas avoir été effectuée. Dans l'introduction du volume qui rend compte de la restauration des lunettes et des cintres, publié en 1992, Perluigi De Vecchi semble vouloir renoncer aux interprétations allégoriques mais, bien qu'il affirme que dans les lunettes il faille «reconnaître le moment le plus avancé et audacieux des inventions techniques et stylistiques de l'ensemble», il n'avance aucune hypothèse d'interprétation à même de rendre compte des implications sémantiques des choix stylistiques décrits, mis à part un vague renvoi à « une inspiration de la réalité » pour une partie des lunettes ${ }^{14}$.

La nouveauté la plus importante au niveau de l'interprétation iconologique rendue possible par la restauration est due, à mon avis, à Barbara Wisch qui révèle, dans un article de 2003, l'existence d'un cercle jaune sur le bras du personnage masculin peint à 
côté du nom d'Aminadab (fig. 2$)^{15}$. J'ai ensuite remarqué deux autres signes, équivalents féminins du signum: deux brassards jaunes portées au bras par deux des femmes des cintres triangulaires (fig. 4 et 5 ).

\section{Fig. 4}

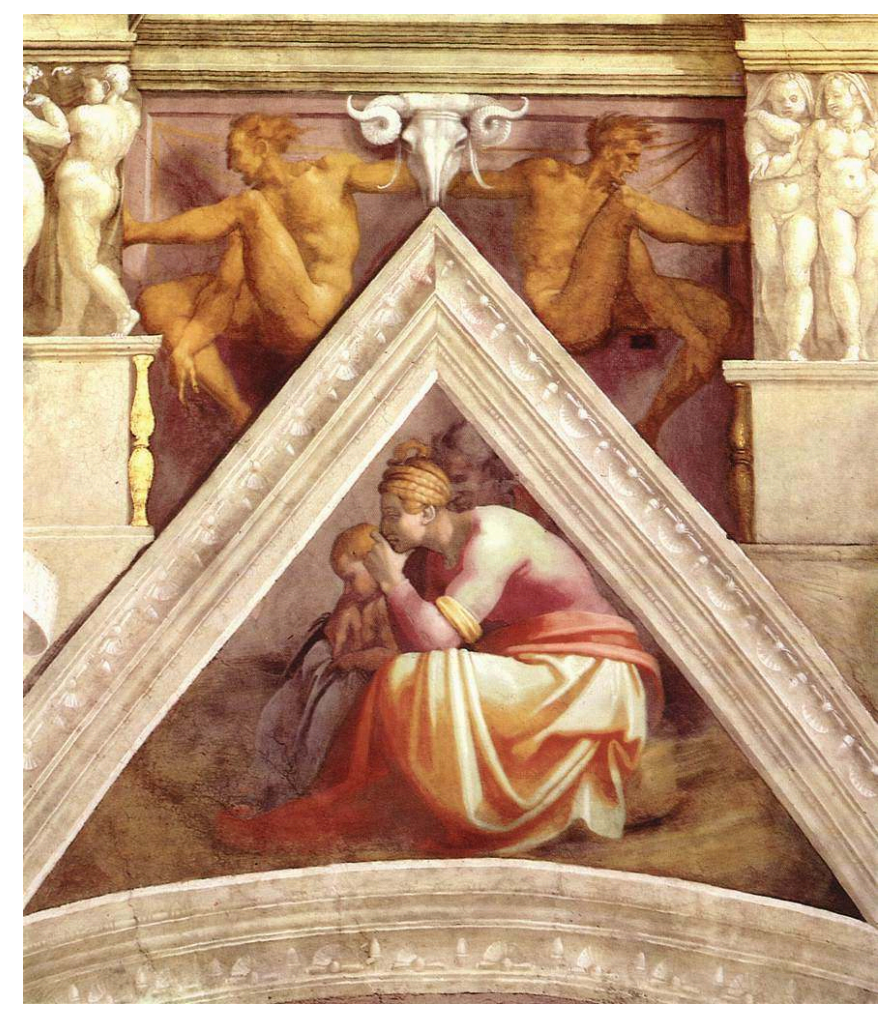

Michel-Ange, Ancêtres du Christ, 1508-12, Chapelle Sixtine Rome 
Fig. 5

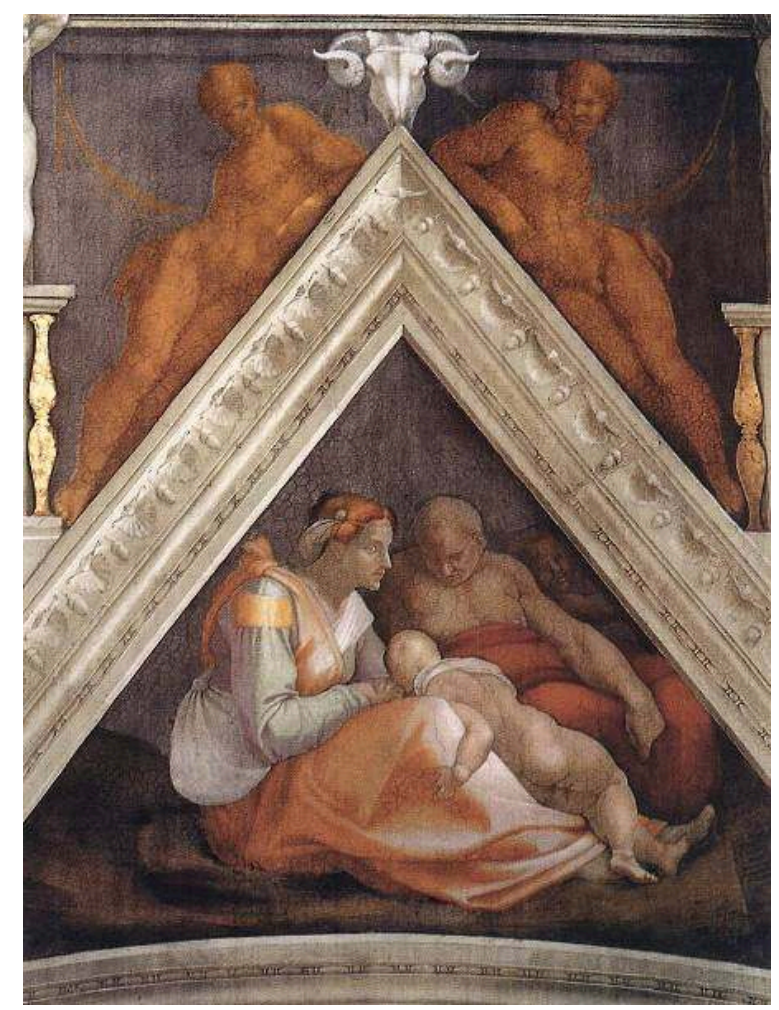

Michel-Ange, Ancêtres du Christ, 1508-12, Chapelle Sixtine Rome

12 Ces signes étaient portés par les juifs dans la Rome papale et dans d'autres villes afin d'éviter les contacts avec les chrétiens et en particulier, selon les textes, les relations sexuelles entre les hommes juifs et les femmes chrétiennes. Il s'agit de signes de ségrégation infamante, comme le prouvent les tentatives, quelquefois réussies, des juifs les plus riches de s'en débarrasser par des taxes ou des faveurs particulières ${ }^{16}$.

La présence du signum a conduit Barbara Wisch à déduire l'appartenance du personnage de la lunette qui porte le nom de Aminadab à l'époque de Michel-Ange. Le signe d'exclusion de la communauté chrétienne qu'il porte doit être compris, d'après elle, par rapport à la ségrégation humiliante que subissait la communauté juive romaine de l'époque. Cette interprétation n'est pas totalement convaincante si l'on considère que le signum était souvent utilisé par les peintres de la Renaissance, surtout les peintres nordiques, pour "marquer» les figures de l'époque du Christ et en particulier les bourreaux de différents épisodes de la Passion. Cependant, le cercle jaune reste un signe infamant et l'article de Barbara Wisch a le mérite d'avoir ouvert une nouvelle perspective de travail qui nous invite à associer ce signum à d'autres traits typiquement anti-hébraïques pour essayer d'en comprendre le rôle dans le dessein historique et théologique de la Chapelle Sixtine. Le signum ouvre par ailleurs une question féconde quant à l'historicité des Ancêtres. Si nous ne pouvons pas les assigner à l'époque de Michel-Ange, nous ne pouvons pas non plus exclure cette possibilité. Le signum est donc l'un des opérateurs qui contribue à intensifier la "plasticité » temporelle et historique des familles : peuvent elles appartenir en même temps à une époque très ancienne et au temps de « maintenant »?

Pour essayer de réponde à cette question je commencerai par m'interroger sur le statut de ces figures de l'altérité en cherchant à en comprendre le rôle et le sens à partir d'une 
vision qui relie les traits de leurs figures inhabituelles au projet d'ensemble de la chapelle. La Chapelle Sixtine est une véritable "fabrique de l'histoire ", sa localisation et ses fonctions liturgiques en font un lieu de fondation essentiel. C'est seulement dans ce cadre qu'il dévient possible de rendre compte de la façon dont les familles des Ancêtres participent d'un projet idéologique général: celui qui "administre " la question du corps et de la chair en relation avec l'eschatologie chrétienne du salut et qui s'incarne ici dans un construction visuelle de l'histoire d'une grande complexité. Il s'agit alors de se demander si l'inertie de ces figures peut être appréhendée en rapport avec la gestualité héroïque des corps qui donnent forme à l'histoire du salut chrétien telle qu'elle se met en scène sur la voûte et dans le Jugement dernier. La pesanteur et la "mélancolie » qu'expriment les corps las des ancêtres sont, en effet, des valeurs négatives nécessaires pour dessiner le bord extérieur d'un dispositif qui règle dans la théologie chrétienne le rapport à une altérité hébraïque à la fois exclue et fondatrice, irréductible et nécessaire. L'histoire interprétative que j'ai présentée introduit, en effet, tout du moins en filigrane, la "stratification » de l'altérité juive, tantôt historique, tantôt théologique, tantôt «typologique » des Ancêtres de Michel-Ange. Mon hypothèse est donc que dans ces figures se condensent tous les éléments qui résistent au grand projet eschatologique de l'histoire chrétienne. La "réabsorption» de ces forces d'inertie est l'un des objectifs de la théologie du salut, qui l'a conceptualisé à travers la notion de chair, dont saint Paul se sert justement pour définir l'altérité juive : celle d'une vie secundum carnem. Les traits charnels du juif sont les résultats d'une longue élaboration; ils "traversent " les figures des Ancêtres en posant le problème de l'entrelacement des différents niveaux, ce qui est sans doute le défi majeur de ce travail mais également le plus prometteur.

Le corps abandonné d'Aminadab qui arbore en évidence sur sa casaque le signum jaune est-il celui d'un juif romain de l'époque de Michel-Ange, celui d'un «Ancêtre du Christ» qui vit avant la révélation, ou celui qui incarne le trait théologique de "résistance obtuse ${ }^{17}$ " des juifs qui, après l'incarnation, s'obstinent à attendre leur messie ? Son corps est l'objet d'une opération figurative qui mobilise tous ces éléments. En effet, plutôt que de poser la simple question de la «temporalité historique » des Ancêtres, je vais aborder la complexe configuration qui résulte de la rencontre entre le caractère historique de l'altérité juive - dont les marques sont évidentes chez certains des personnages - et le statut théorique qu'elle assume dans la mesure où elle participe à un paradigme théologique, aussi bien par son caractère "typique " et donc anhistorique, que par sa position rhétorique ou herméneutique, quand l'altérité juive fonctionne comme un "argument» au travers duquel s'articule une réflexion des chrétiens sur eux-mêmes ${ }^{18}$.

16 Ces interrogations compliquent et enrichissent la question de l'identité des Ancêtres en leur attribuant un rôle important pour la définition de l'identité chrétienne elle-même. Le juif est l'autre, mais, en tant qu'ancêtre, il est en même temps le " même ». Inclusion et exclusion s'affrontent en produisant une tension qui détermine la définition visuelle de la généalogie du Christ. De cette parenté ancienne et prestigieuse, le christianisme veut capter l'enracinement historique et légitimant, alors qu'en même temps, il doit distinguer son projet œcuménique du modèle généalogique, dans la mesure où la doctrine chrétienne propose un modèle d'appartenance radicalement nouveau.

La figure du juif en tant qu'ancêtre est une catégorie de la "théologie de l'histoire " impliquée par l'ensemble des fresques de la Chapelle Sixtine. Le rapport entre l'altérité 
juive et la "vie concrète» se joue au niveau de cette définition catégorielle, sans impliquer aucune forme naïve de "réalisme documentaire ", comme le prétendait l'histoire de l'art du XIX $x^{e}$ siècle. Pour faire apparaitre les dispositifs idéologiques qui prennent appui sur l'historicité complexe des Ancêtres je vais procéder à l'étude des schémas iconographiques sous jacents à la figuration de ces étranges familles, suivant l'hypothèse que la dimension " domestique » résulte d'un travail de transformation ou "d'altération" de schémas iconographiques familiaux préexistants. On peut ainsi comprendre l'aspect humble des Ancêtres comme un « abaissement » dû à l'altération du schéma « noble » de la Sainte Famille.

Pour comprendre la position des figures des Ancêtres dans le dessin global de la Chapelle Sixtine, commençons par prendre en considération le trait topologique qui leur confère un statut liminaire (fig. 6). La première observation est d'ordre tectonique : sur quoi « reposent » les lunettes et les cintres et à quoi servent-ils, à leur tour, de support?

Fig. 6

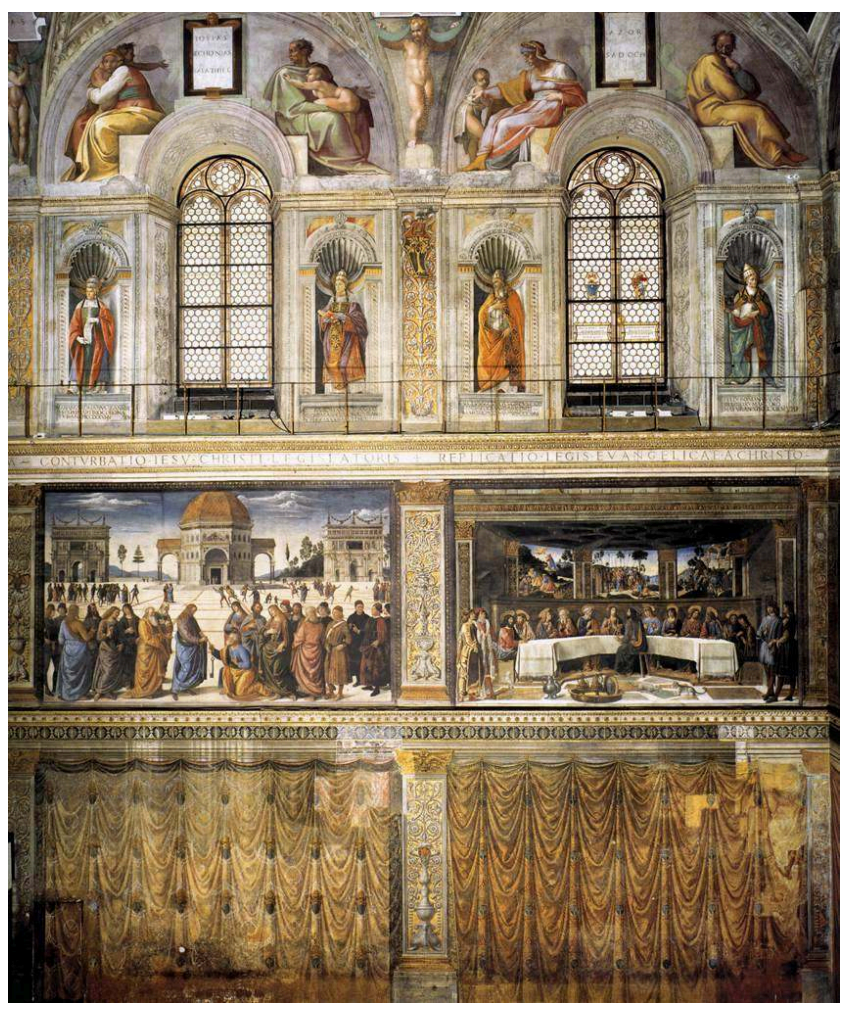

Parois de la Chapelle Sixtine : histoires de la vie du Christ, Premiers papes et Ancêtres du Christ

La poussée verticale que les figures hiératiques de la série des Premiers Papes exercent vers le haut n'est pas relancée par les lunettes, au contraire, elle est amoindrie et écrasée par la pesanteur des figures affaissées des Ancêtres. Leur "poids visuel ", comprimé dans les lunettes et dans les cintres, accentue, par contraste, la poussée de la voûte relancée (?) par les figures des Sibylles et des Prophètes. La transition est assurée par des nus couleur bronze qui, dans certains cas, miment les gestes d'abandon des Ancêtres, et dans d'autres ont une posture inverse, proche de l'agitation extrême (fig. 7) 
Fig. 7

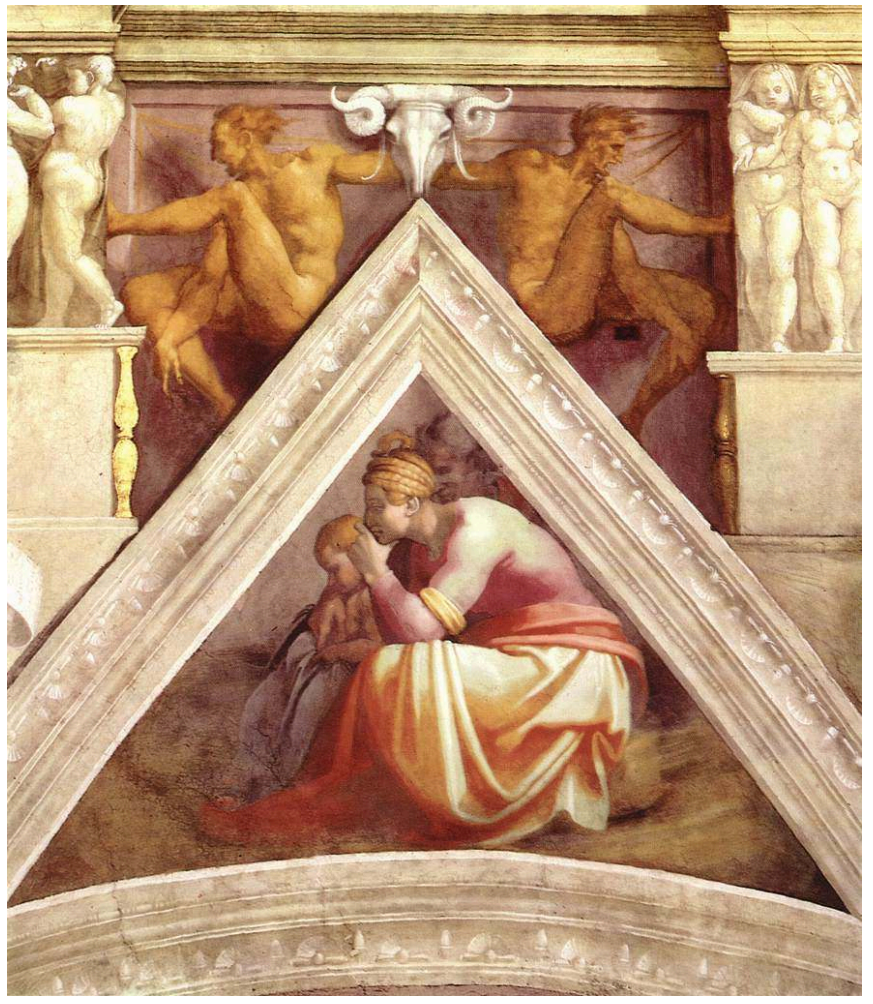

Michel-Ange, Ancêtres du Christ, 1508-12, Chapelle Sixtine Rome

20 Le jeu de force qui tend le rapport entre les lunettes et les figures qui occupent les espaces proches du cadre de la voûte sert en fait de matrice énergétique pour un type de contraste qu'on pourra justement caractériser comme stylistique, si l'on veut définir par ce terme la «hauteur» du registre discursif adopté. Les Sibylles et les Prophètes incarnent des types "héroïques" alors que les ancêtres sont présentés sous des modalités stylistiques « humbles ». La proximité des deux registres stylistiques opposés a un effet d'intensification : à côté de l'inspiration héroïque des Prophètes et des Sibylles, les familles des ancêtres apparaissent encore plus prosaïquement absorbées dans leurs existence humble et répétitive.

Fig. 8

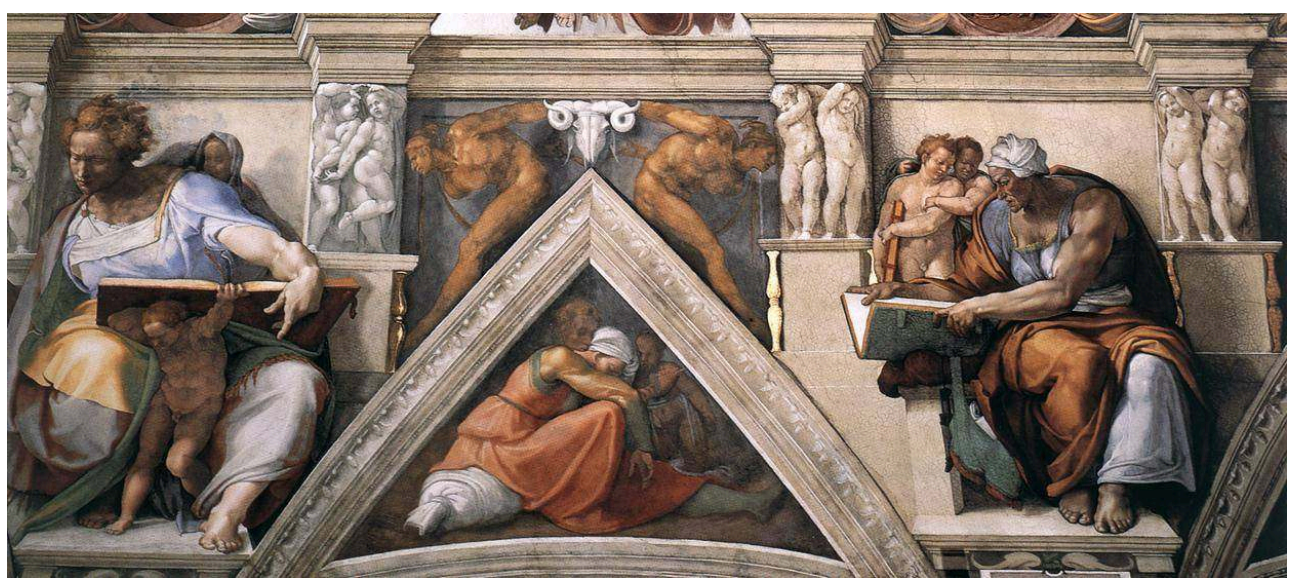

Voûte de la Chapelle Sixtine 
21 distinctif du style expressif des saintes écritures et particulièrement des évangiles. A ce propos, il cite un passage du De Doctrina cristiana dans lequel saint Augustin parle de la Scripturarum mirabili altitudine et mirabili humilitate ${ }^{19}$. Saint Augustin y défend le sermo umilis des évangiles qui correspondrait au «style » de vie du Christ «modèle de vie et de mort saintes et sublimes vécues comme une vie ordinaire ou comme les scènes d'une comédie vécue parmi les humilis personae qui ont été ses premiers disciples [...]. Mais le Christ lui-même, et pas seulement le milieu qui l'entoure ou son sort sur la terre, exprime l'antithèse entre l'humble et le sublime sous sa forme la plus aiguë et passionnante : et alors, il ne s'agit plus du discours, mais des faits » ${ }^{20}$.

Pour Auerbach la Divine Comédie de Dante est l'exemple majeur de ce registre stylistique mixte, traversé par une tension interne qui en accentue la valeur expressive. Du point de vue de la rhétorique classique - écrit Auerbach - dans le poème de Dante : «il y a trop de réalisme, trop de vie concrète, trop de biotikon comme disaient les théoriciens grecs, tant dans les paroles que dans les faits et non seulement chez les habitants des Enfers, mais aussi du Purgatoire et souvent même du Paradis " ${ }^{21}$. Le jeu de contraste entre le sublime et le biotikon dans la voûte de la Chapelle Sixtine définit une sorte de « réalisme » qui résulte d'un vigoureux jeu de contrastes formels et sémantiques, et ce, non seulement grâce à la présence des éléments de la "vie concrète " (fig. 8). La référence à la texture stylistique de la Divine Comédie que Michel-Ange « avait presque entièrement à l'esprit » et qu'il étudiait comme un poète quasi-professionnel, nous invite à affiner notre regard sur le biotikon du cycle des Ancêtres ${ }^{22}$. Si, en effet, l'on reconnaît dans la Voûte un "discours visuel mixte" comparable à celui du poème dantesque, on doit alors essayer de comprendre la valeur de l'humilitas de ces figures comme toujours potentiellement réversible ou «sublimable». En d'autres termes, reconnaître l'appartenance de la dimension réaliste des figures des Ancêtres à une tradition stylistique chrétienne et dantesque nous permettra d'éviter une évaluation simplifiée et univoque de leur « humilité » ainsi que de leurs traits anti-hébraïques.

Steinman le premier a observé que la séquence chronologique des noms des ancêtres ne procède pas linéairement paroi après paroi, mais zigzague entre une paroi et l'autre parallèlement à la série chronologique des Premiers Papes, située en dessous et elle aussi disposée selon le même ordre ${ }^{23}$. Ce parallélisme entre les deux séries est confirmé par certains traits de continuité chromatique et correspondrait, au niveau idéologique, à une intention commune de légitimation du pouvoir papal et de la primauté du pape ${ }^{24}$. En effet, les ancêtres du Christ sont également les "ancêtres des papes ", par leur ancienneté ils apportent aux papes un supplément d'autorité ${ }^{25}$, enracinant leur fonction sacerdotale dans l'antiquité des origines, en conformité avec ce qui se produit dans la relation entre la série des papes et les fresques $d u X V^{e}$ siècle de la Chapelle Sixtine dans lesquelles on raconte les «vies institutionelles et sacerdotales» de Moïse et Jésus ${ }^{26}$. Un commentateur a d'ailleurs décrit le groupe des premiers papes sur les parois comme un "arbre généalogique ${ }^{27}$. Toutefois, si le lien de continuité entre les Papes et les Ancêtres est effectivement suggéré par le jeu des couleurs et des gestes, on ne doit pas oublier que la généalogie des papes est une généalogie sui generis puisqu'elle ne se base pas sur la transmission par le sang mais sur le passage d'une charge élective. Le trait commun entre le cycle des Papes et celui des lunettes et des cintres est le partage d'une conception du temps historique fondé sur le principe "génératif » qui distingue chaque nouvelle génération par le nom d'un ancêtre d'abord et d'un pape

Images Re-vues, 9 | 2011 
ensuite. Le nom du pape intègre, par ailleurs, chacun des individus dans une liste qui les transcende, la séquence étant le principe de continuité qui fonde la pérennité de la fonction.

Dans la superposition entre la généalogie charnelle des ancêtres et la généalogie élective des papes s'exprime bien une continuité légitimante, mais aussi la différence entre la façon « antique et juive » de transmettre l'autorité au sein de la lignée et celle "nouvelle et chrétienne », qui ne peut pas s'appuyer sur une logique de descendance familiale. La contiguïté dans l'espace de la Chapelle Sixtine affirme la continuité entre les deux "généalogies " alors que la discontinuité et la différenciation s'expriment à travers le contraste qu'opposent les attitudes hiératiques et dignes des Papes à celles lasses et domestiques des Ancêtres. Le passage entre le modèle de parentèle tribale et ethnique du "temps juif antique " et le modèle nouveau et chrétien de "parentèle spirituelle » constitue l'un des nœuds décisifs pour comprendre les figures des Ancêtres.

\section{De la généalogie charnelle à la parenté spirituelle}

Un nom propre désigne, en principe, un seul individu, pour certains, il s'agit d'un " désignateur rigide ", c'est-à-dire d'un type de signe très pauvre parce que limité à une seule opération référentielle exempte d'ambiguïté dite, justement, de dénomination. En Occident, depuis l'époque de la Grèce archaïque, quand un nom propre entre en relation avec l'effigie d'un individu, une opération d'individuation se produit qui a une valeur cultuelle de substitution de l'absent, particulièrement efficace dans le contexte des rites funéraires et politiques. En vertu de cette mémoire culturelle et rituelle millénaire, la dénomination de l'effigie s'effectue de façon quasi automatique; ainsi d'un portrait de César on dira "c'est César » et non " c'est une image de César " ${ }^{28}$.

Les caractéristiques proprement plastiques et figuratives de l'effigie passent alors au second plan à tel point que pour en appréhender la forme, il devient nécessaire de "suspendre » le pouvoir du nom. Une des tentations des historiens de l'art qui se sont occupés du cycle des Ancêtres du Christ a été au contraire de ne se fier qu'au pouvoir du nom, en réduisant la complexe ambiguité des images à l'univocité rigide des noms inscrits en capitales dans les cartouches placées au centre de chaque lunette. Si je dis que le personnage assis à côté du nom d'Aminadab est «Aminadab » je peux chercher dans la Bible, ou ailleurs, le passage où son nom apparaît, raconter les histoires dont il a été le protagoniste, lui construire finalement une identité textuelle en grande partie indépendante de l'aspect effectif du personnage et encore plus du contexte d'images dans lequel il s'insère. L'impossibilité de rapporter avec certitude les noms aux personnages masculins quand il y a trop de candidats dans les lunettes ou, au contraire, pas assez, n'a cependant pas gêné les spécialistes, au point que certains ont même voulu donner un nom à certains enfants non encore nés, mais supposés présents dans le ventre de leur mère.

En général, et bien au-delà du cas spécifique de la Chapelle Sixtine, la dénomination a souvent été considérée comme l'objectif ultime du travail de l'iconologue comme si, une fois nommé, le personnage était finalement libéré de son statut inquiétant et polysémique d'image pour être ancré dans un statut linguistique et identitaire assuré. Les Ancêtres ont été ainsi recouverts et cachés en tant qu'images par les noms qui les accompagnent, à tel point qu'à la fin, on a renoncé à les regarder : "on n'y voit rien " comme aurait dit Daniel Arasse ${ }^{29}$. Les interprétations d'Edgar Wind et de Frederick 
Hartt que j'ai évoqués plus haut sont une version raffinée de la même pulsion dénominative : insatisfaits par la pauvreté sémantique des noms, les deux historiens de l'art ont réussi à les transformer en mini-histoires grâce à la traduction des noms hébreux en latin. Le point faible de cette tentative est d'une part la disproportion entre la rigidité univoque du nom et la riche ambiguïté des figures et, d'autre part, l'absence de critères de pertinence capables de déterminer le choix de l'une ou de l'autre des minis histoires bibliques possibles. Il me faut cependant rappeler que l'exégèse fondée sur la traduction des noms hébreux n'est pas une invention d'Edgar Wind et de Frederick Hartt puisqu'elle repose sur une tradition qui remonte jusqu'aux Pères de l'Eglise et plus particulièrement à Origène et à saint Jérôme qui l'appliquent en général pour tisser des liens typologiques ou allégoriques entre l'Ancien et le Nouveau Testament. Dans le contexte de renouvellement des études hébraïques caractéristique des milieux humanistes proches de la cour de Jules II, cette pratique interprétative était bien connue, si bien qu'en principe, il est possible qu'on en trouve trace dans la Chapelle Sixtine. Cependant, cette séduisante hypothèse d'une iconographie dérivée de la traduction des noms doit être abandonnée face à l'impossibilité évidente de l'appliquer sans faire violence à l'aspect visuel des figures. Un exemple peut servir à mieux éclaircir ce point : il s'agit d'un autre cas tiré de l'analyse d'Edgar Wind, celui de Ioatham qui se traduit par perfectus ou consummatus « Nihil enim ad perfectum adduxit lex » comme l'écrit saint Paul dans la lettre aux Hébreux (VII, 19), dirigeant cette accusation de présomption contre les sacerdoces lévites auxquels Ioatham appartient.

Fig. 9

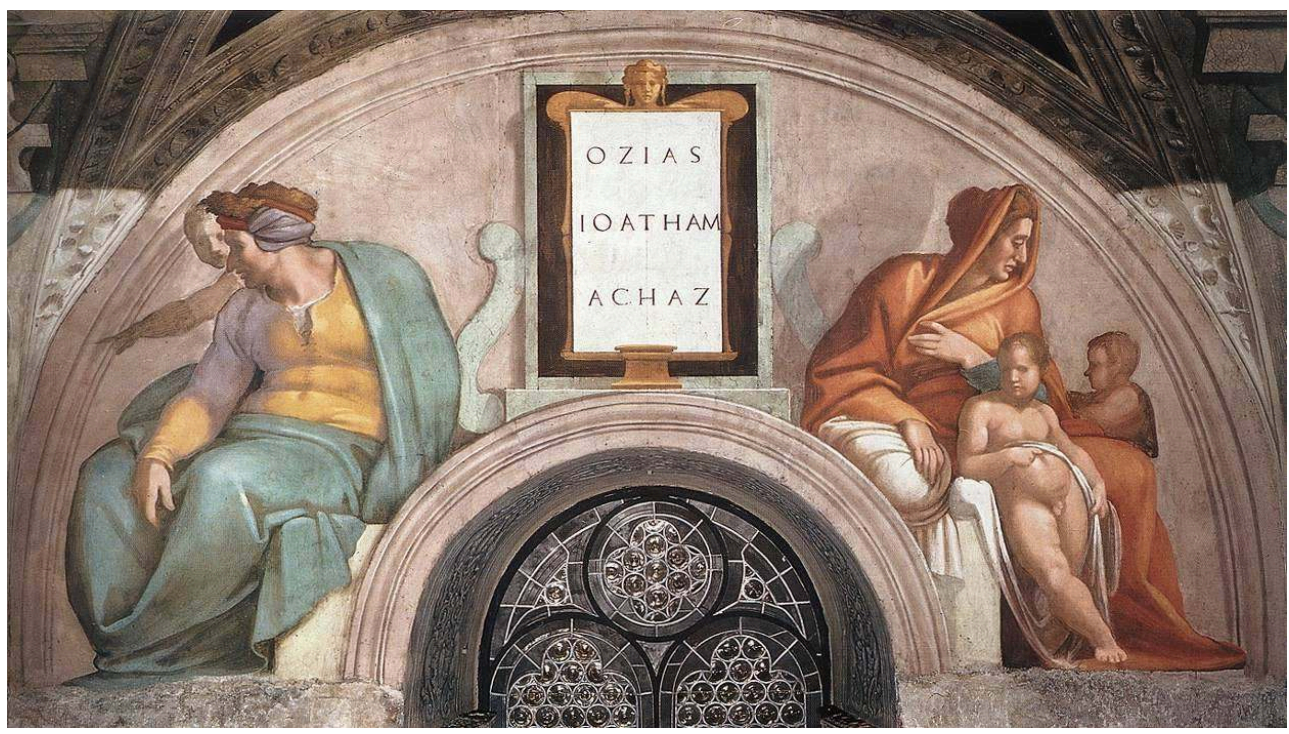

Michel-Ange, Ancêtres du Christ, 1508-12, Chapelle Sixtine Rome

Le personnage peint à la gauche de la lunette, à côté des noms Ozias, Ioatham et Achaz est non seulement identifié comme étant Ioatham (fig. 9), mais est également décrit, à partir du texte paulinien, comme « plein de ressentiment à cause du manque de respect dû à ses prérogatives ${ }^{30}$. La domination du registre verbal non seulement oriente et détermine la sélection des traits figuratifs présentés par l'image mais elle leur attribue une valeur affective aux connotations morales, très affirmée dans le contexte textuel de la « source », mais loin d'être évidente dans la figure elle-même. 
Alors que nombreux sont ceux qui se sont lancés dans le jeu des noms, peu se sont interrogés sur la fonction et sur la structure de la liste généalogique comme forme culturelle dotée d'une façon propre de signifier. Lisa Pon fait exception à la règle. En 1998, elle a suggéré que les noms des lunettes ne fonctionnent pas comme des dénominations d'individus, mais avant tout comme éléments d'une série ${ }^{31}$. Evidemment, le passage du temps est biologiquement mesuré par les générations et marqué par ces noms, mais ce qui compte dans la généalogie de Jésus-Christ, c'est la chaîne qui relie Jésus à Abraham et à David à travers l'énumération et le lien des noms entre eux plutôt que chaque nom isolé. Tous les noms doivent donc être pris ensemble pour dénoter les Ancêtres du Christ. De la même façon, toutes les figures, hommes et femmes, jeunes et vieux devraient être vus - selon l'auteur - comme un ensemble identifiable aux Ancêtres du Christ, même s'il est impossible de donner à chaque personnage une identité individuelle. De façon cohérente, cette lecture se conclut par l'affirmation que «la généalogie verbale vise directement le but théologique de la série : l'Incarnation du Christ ». Cette analyse permet une avancée certaine puisqu'elle attribue à la forme de la liste un pouvoir de signification autonome par rapport à la simple fonction de dénomination de chaque ancêtre. Pourtant l'Incarnation du Christ est justement le point de rupture de la logique généalogique de la transmission du pouvoir patriarcal et de la prêtrise par la voie du sang. Il me semble donc nécessaire d'étendre la réflexion à partir d'une interrogation sur la relation entre la logique sérielle de la liste verbale et la logique spatiale des lunettes et des cintres. A la différence de ce qui se passe pour les Ancêtres du Christ placés sur les cadres en ogive des portails gothiques de Laon, Chartres et Sienne (fig. 10), l'organisation en lunettes et cintres se prête mal à accueillir la linéarité de la liste.

Fig. 10

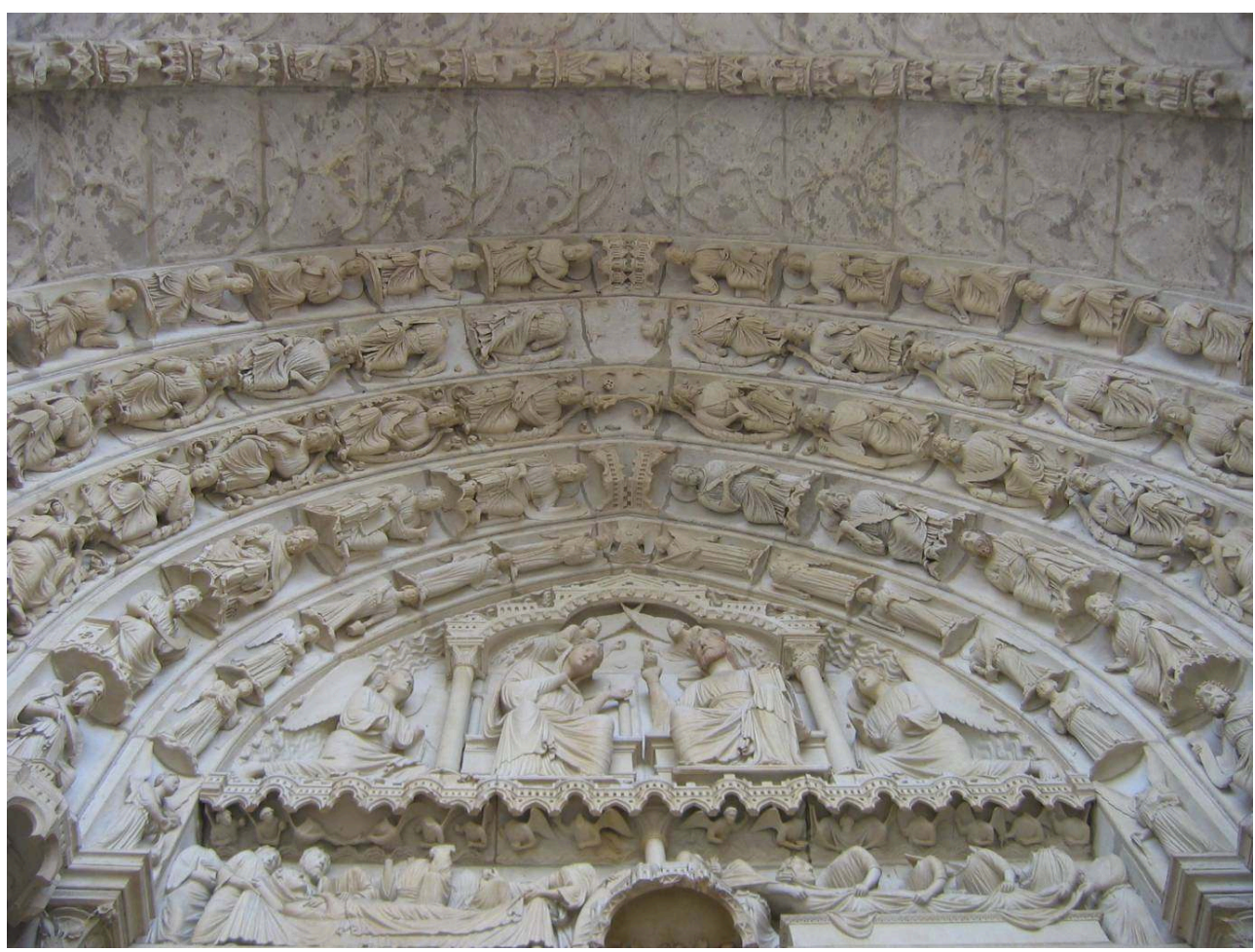

Portail nord de la Cathédrale de Chartres 
Dans ces exemples gothiques, la logique sérielle de la succession résulte d'une contamination avec la forme de l'arbre de Jessé, diagramme qui utilise l'axe vertical pour signifier l'antériorité et la puissance germinale de la ligne généalogique. La forme en bande qui, selon Gilbert, aurait été inventée par Giotto dans la Chapelle de l'Arena a, elle aussi, un développement linéaire marqué, puisque les quarante et un bustes se succèdent sur des bandeaux linéaires situés autour des histoires de Marie et de Jésus. Le développement horizontal des familles des Ancêtres de Michel-Ange les situe, par contre, toutes sur le même plan tandis qu'aucun élément vestimentaire ne les distingue qui permettrait de les situer dans une époque plus ancienne ou plus récente. Le seul élément séquentiel est constitué par la série des noms, placés pourtant de façon irrégulière dans les cartouches par groupes de deux, trois ou quatre noms, voire quelquefois d'un seul. La succession de plusieurs noms dans le cartouche est brève, la série est sans cesse interrompue et fragmentée en microlistes placées dans un champ spatial délimité par le cadre, tandis que le parcours zigzaguant, qui oblige à passer d'une paroi à l'autre pour respecter l'ordre des noms de la généalogie de Matthieu, trouble ultérieurement le séquençage des figures.

Fig. 11
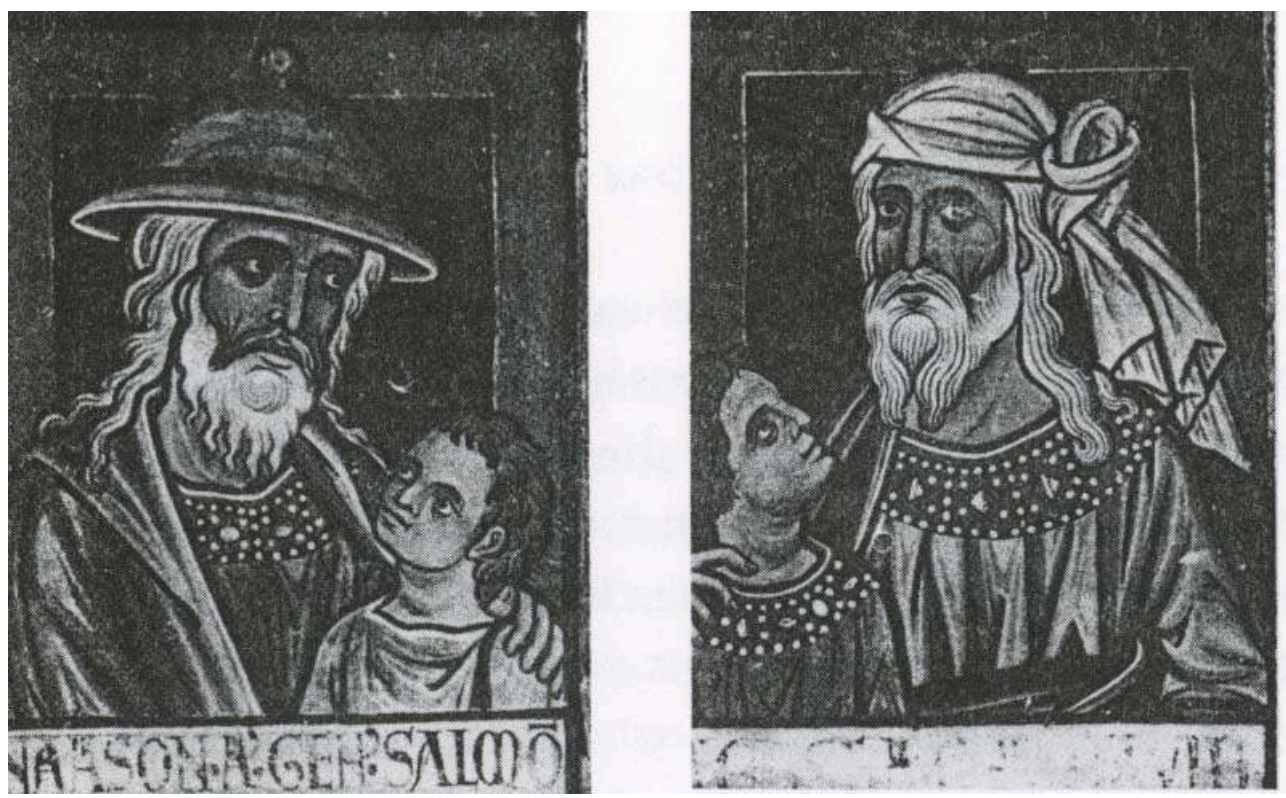

Ancêtres du christ, $X I^{e}$ siècle (détruit) voûte de la cathédrale de Sigena

31 Le principe génératif qui relie un ancêtre à son fils ou un roi à son descendant, comme c'était le cas dans la cathédrale espagnole de Sigena (fig. 11) ou sur le portail de Chartres, est en plus remplacé, dans la Chapelle Sixtine, par une iconographie du noyau familial : il est donc impossible de déterminer qui assume le rôle de l'ancêtre, surtout lorsqu'il y a deux ou trois enfants. La transmission charnelle est effectivement exprimée grâce à l'insistance sur l'allaitement et sur les soins portés aux nombreux enfants, mais cette dimension est déconnectée du séquençage hiérarchisé des séries généalogiques. On peut donc conclure que la distribution horizontale des lunettes et des cintres va à «l'encontre » de la hiérarchie temporelle « verticale » impliquée par la série des noms et qui est ici continuellement suspendue et interrompue. 
Fig. 12

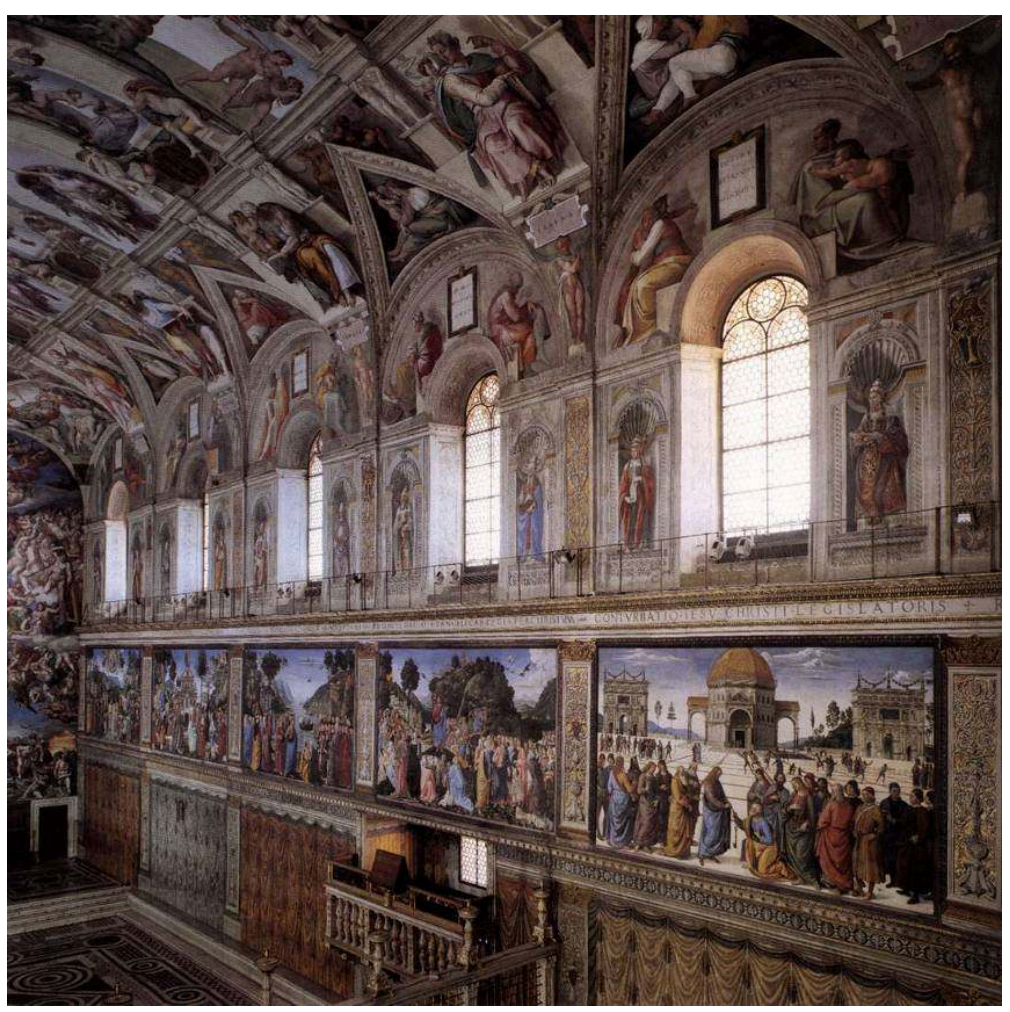

Chapelle Sixtine

Si l'on considère que les lunettes et les cintres qui abritent les figures des Ancêtres respectent un principe de contiguité plutôt que de succession, on peut se demander avec Gilbert et Dotson - s'il ne s'agit pas de représentants, contemporains entre eux, d'une seule génération plutôt que de représentants des temps distincts entre eux des quarante générations égrenées par les noms des Ancêtres du Christ (fig. 12). On ne pourra revenir sur cette hypothèse qu'après avoir éclairci la fonction théologique et en même temps anthropologique de la "généalogie du Christ " qui ouvre l'évangile de Matthieu. Le Liber generationis Jesus Christi est en effet l'un des passages les plus problématiques de cet évangile puisqu'il renferme un paradoxe qui, en même temps, utilise et met en crise le principe généalogique comme le résume bien saint Augustin lorsqu'il observe: «l'énumération des Ancêtres du Christ arrive à Joseph alors que Jésus n'est pas le fils de cet homme mais de la Vierge Marie $»^{32}$.

Si l'on se situe, en d'autres termes, dans une perspective d'anthropologie de la parenté et que l'on s'interroge sur les critères qui président le passage du nom et du pouvoir entre père et fils on constate que l'intervention de Dieu dans l'Incarnation et la naissance de Jésus provoquent une rupture, qui annule définitivement la valeur de la transmission par le sang propre à la généalogie charnelle, ainsi que la façon dont elle organise le cours de l'histoire. Ce n'est pas un hasard si la liste des ancêtres du Christ se trouve, au début du livre de Matthieu, précisément à l'endroit où l'auteur doit gérer l'articulation entre l'époque de l'Ancien Testament et celle du Nouveau. Plaçant Joseph et Jésus dans la lignée de David, le texte affirme la légitimité royale du Christ et lui donne le rôle décisif de Messie venu accomplir les prophéties bibliques. Dans les pages suivantes, toutefois, les modalités généalogiques de la transmission de la légitimité de père en fils selon le lien du sang sont ébranlées par le récit de l'Annonciation. 
L'Incarnation substitue à la filiation patrilinéaire fondée sur le sang une filiation divine qui conserve, cependant, la génération charnelle, par le biais de l'intervention bien humaine de Marie. Le lien du sang est pourtant remplacé par une forme de parenté alternative: une parenté spirituelle qui lie le fils à un père divin. Jésus doit donc prendre place dans un système patriarcal de transmission de la légitimité que lui-même renverse en se proclamant fils de Dieu. Une opération paradoxale s'opère alors sur les Ancêtres du Christ : car d'un côté ils doivent être inclus dans l'histoire chrétienne pour enraciner son programme messianique dans le temps des rois et des patriarches jusqu'à celui originel de la création du premier homme, mais de l'autre ils doivent être " exclus» de l'histoire chrétienne pour souligner la crise profonde du modèle de transmission charnelle dont ils sont les dépositaires. Le modèle généalogique patriarcal fonde l'histoire d'Israël mais il est incompatible avec le prosélytisme universel ouvert à «toutes les nations » caractéristique du christianisme. "Les évangélistes Matthieu et Luc - souligne la théologienne Karin Friis Plum - utilisent la forme généalogique pour démontrer qu'avec Jésus les possibilités de la généalogie sont complètement épuisées. La chrétienté sépare en effet l'identité religieuse du modèle de parenté dominant ${ }^{33}$. La généalogie du Christ joue dans l'Evangile le rôle d'une construction mythologique capable de recomposer la tension qui oppose la jonction avec l'histoire généalogique de l'Ancien Testament, à la discontinuité qui fonde l'histoire anti-généalogique du Nouveau.

Le rapport entre les noms et les figures des Ancêtres dans la Sixtine donne une forme visible à cette relation paradoxale : d'un côté, nous reconnaissons dans la liste des noms ce qui permet l'inclusion de l'histoire chrétienne dans l'histoire des Hébreux et dans l'histoire humaine, dans la mesure où la lignée de David descend du premier homme créé par Dieu, de l'autre nous voyons émerger dans les corps des ancêtres des traits d'altérité qui ont valeur d'autant de marques d'une opération d'exclusion et de rejet de ce qui, dans cette transmission exclusivement charnelle, ne peut s'ouvrir au nouveau modèle d'appartenance chrétienne.

Les cartouches portant les noms des Ancêtres en grandes capitales assument le rôle de valorisation de la généalogie en s'associant aux autres inscriptions en capitales présentes dans la chapelle et en particulier aux tituli qui encadrent les histoires de Jésus et de Moïse représentées dans les fresques du XV siècle. Ce n'est pas un hasard si ces textes latins décrivent les moments les plus importants de l'histoire d'Israël en établissant de manière tout à fait explicite leur valeur de préfiguration de l'histoire de l'Eglise ${ }^{34}$. Sur la paroi des Histoires de Moïse, on lit, par exemple, Promulgatio evangelicae legis per Christum, (Promulgation de la lois évangélique par le Christ); à ce "titre " répond, du côté opposé de la chapelle, le "titre »: Promulgatio Legis scripte per Moise (promulgation par Moïse des lois écrites). Ces textes ont des caractéristiques graphiques et monumentales semblables à celles des noms des Ancêtres et une valeur comparable d'inclusion de l'histoire du peuple d'Israël dans l'histoire chrétienne. Nous pouvons en somme opposer d'un côté la valorisation du modèle généalogique porté par les inscriptions dans les cartouches et de l'autre la construction de la différence et de l'altérité juive dévalorisée dont les figures mêmes sont porteuses. Cette articulation nous conduit à réfléchir sur la "mémoire visuelle" condensée dans les figures des familles des ancêtres, sur les questions anthropologiques qu'elles incarnent, sur les relations avec ce qui, à l'époque de Michel-Ange, constituait les formes historiques de marquage de l'altérité juive. 
Fig. 13

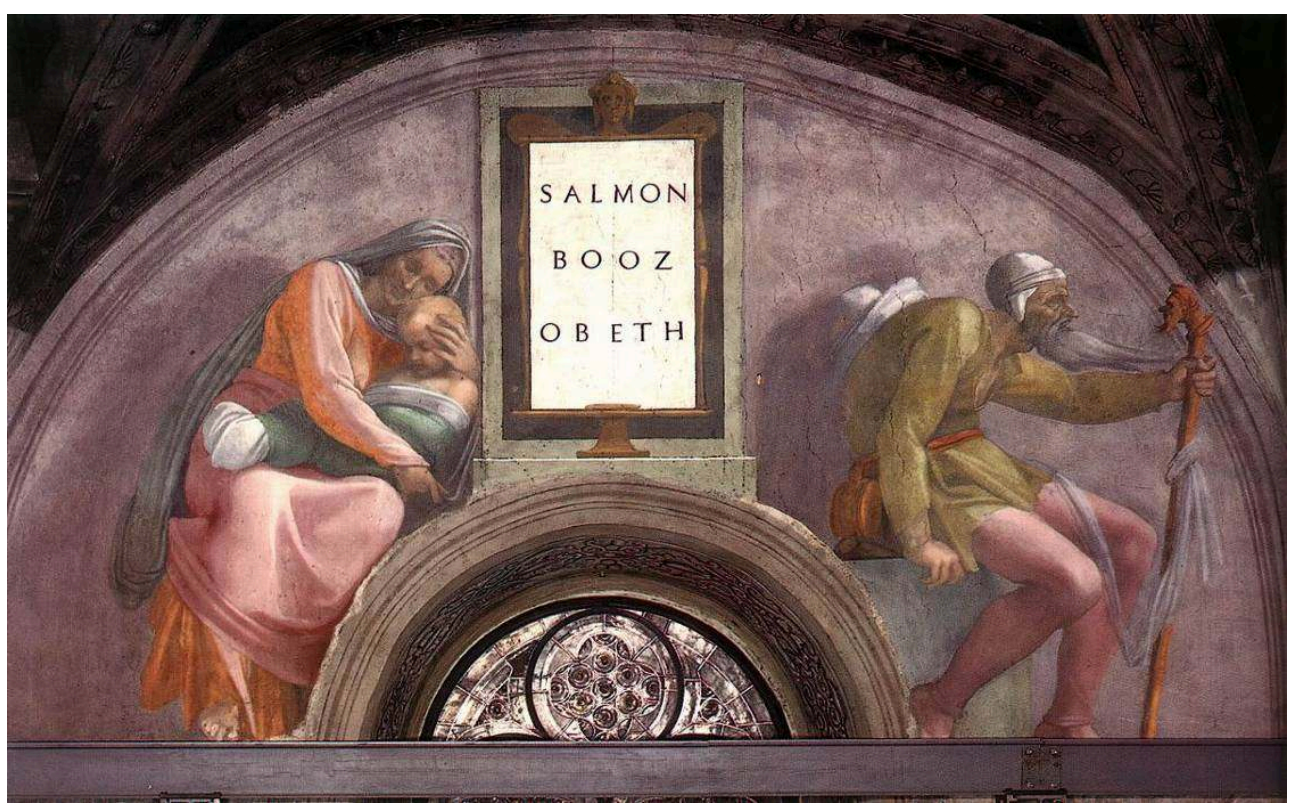

Michel-Ange, Ancêtres du Christ, 1508-12, Chapelle Sixtine Rome

En d'autres termes, le subtil travail d'altération des traits et des attitudes, qui, dans certains cas frôle le grotesque, enregistre le processus de construction d'une altérité nécessaire à opposer une différence à l'identité parentale ou charnelle entre juifs et chrétiens affirmée dans la liste généalogique des noms (fig. 13). Dans la mesure où il sépare le juif du chrétien, le signum infamant arboré par le personnage qui porte le nom d'Aminadab dans la lunette représente l'une des expressions de la différenciation ou de l'altération dévalorisante. D'autres formes d'altération d'ordre figuratif participent à cette opération exclusive qui contredit le discours d'identité d'origine affirmé par les noms.

\section{Secundum carnem}

Un observateur attentif des figures des Ancêtres sera surtout frappé par la dimension proprement iconographique de leur «carnalité", une catégorie que j'emploierai, pour l'instant, dans l'acception du sens commun en tant que relative au corps, aux processus et aux événements purement charnels qui rythment l'existence et son maintien, mais que nous verrons bientôt prendre une acception plus précise et assumer une charge idéologique fondatrice. 
Fig. 14

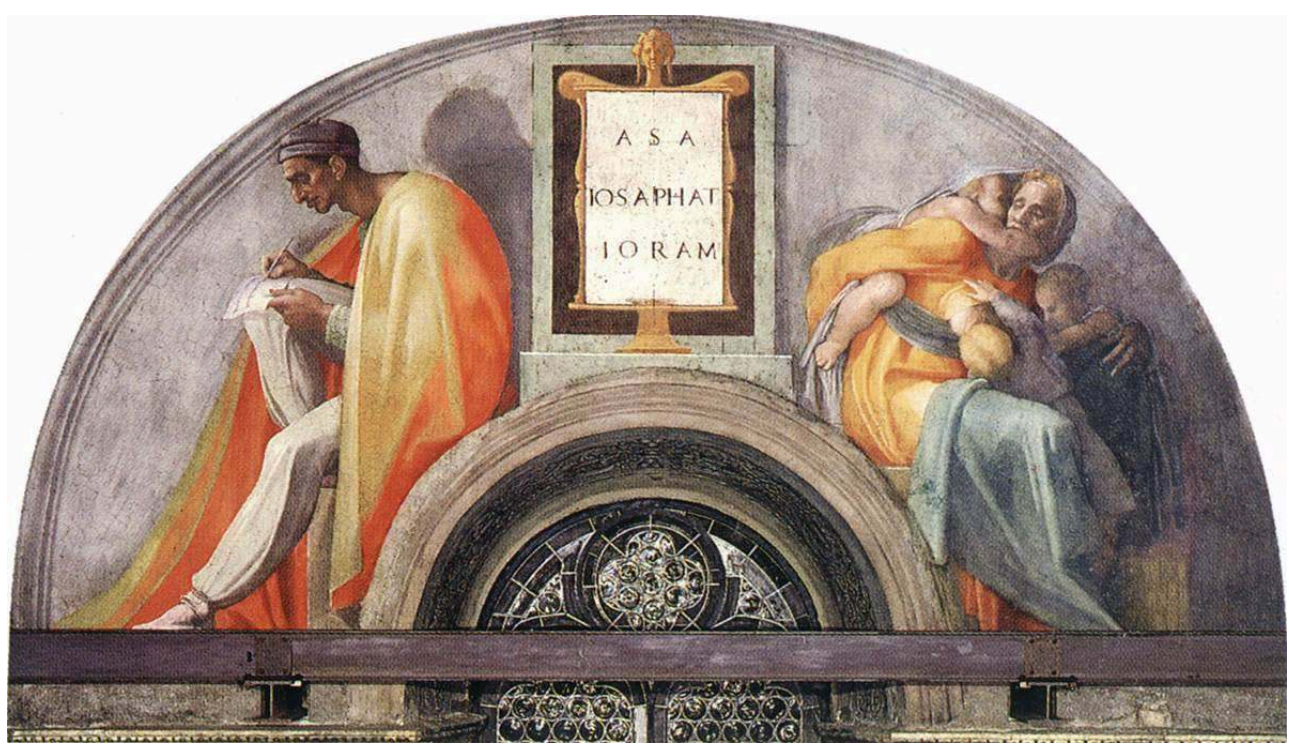

Michel-Ange, Ancêtres du Christ, 1508-12, Chapelle Sixtine Rome

Les voies d'accès à la compréhension de cette dimension sont nombreuses, mais la "mémoire visuelle" qui semble se réactiver, comme en négatif, dans l'incongruité des figures et des familles qui peuplent les lunettes et les cintres, y joue un rôle fondamental. Cette incongruité doit d'abord être renvoyée à l'ensemble des schémas iconographiques qui ont, au cours des siècles et jusqu'à l'époque de Michel-Ange, joué un rôle dans la représentation du groupe familial par antonomase, celui de la sainte Famille (fig. 15). 
Fig. 15

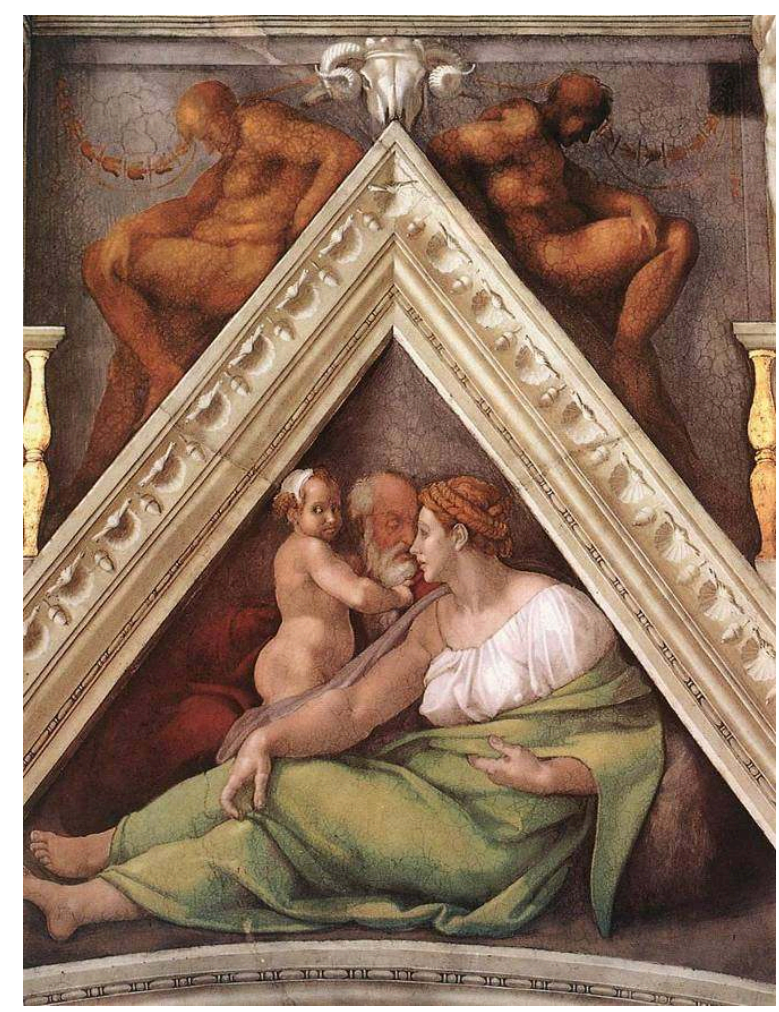

Michel-Ange, Ancêtres du Christ, 1508-12, Chapelle Sixtine Rome

39 Face à ces femmes qui bercent les enfants, les allaitent et les soignent; face aux familles où on aperçoit un vieux père derrière des mères avec enfants notre mémoire visuelle convoque immédiatement la Nativité et l'Adoration des mages. La représentation de ces épisodes est, en effet, le domaine privilégié où s'articule le passage entre l'ancienne généalogie exclusivement charnelle et le moment inaugural de la Nouvelle Ere. Il est donc intéressant d'observer que, alors que dans la figure de Marie - dont le corps devient, avec l'Annonciation, le dépositaire du mystère d'une génération en même temps divine et charnelle - se manifeste toujours la conscience de la divinité du fils, le père " adoptif » Joseph assume souvent un rôle marginal. Joseph est souvent représenté comme mélancolique, assoupi, ou occupé à des travaux domestiques. Comme l'a révélé Ruth Mellinnkoff, le mari de Marie, représenté avec des traits juifs stéréotypés, est souvent situé à la marge de l'espace où se trouvent la Vierge et l'enfant ${ }^{35}$. Cette position liminale est la manifestation visuelle d'une " position théologique » complexe liée au "dilemme» de Joseph, et par extension au manque de reconnaissance de l'identité divine de Jésus de la part des juifs. Mellinnkoff établit un lien entre la position liminale de Joseph et sa posture mélancolique, toutes deux liées à sa perplexité face à la divinité de l'enfant. 
Fig. 16

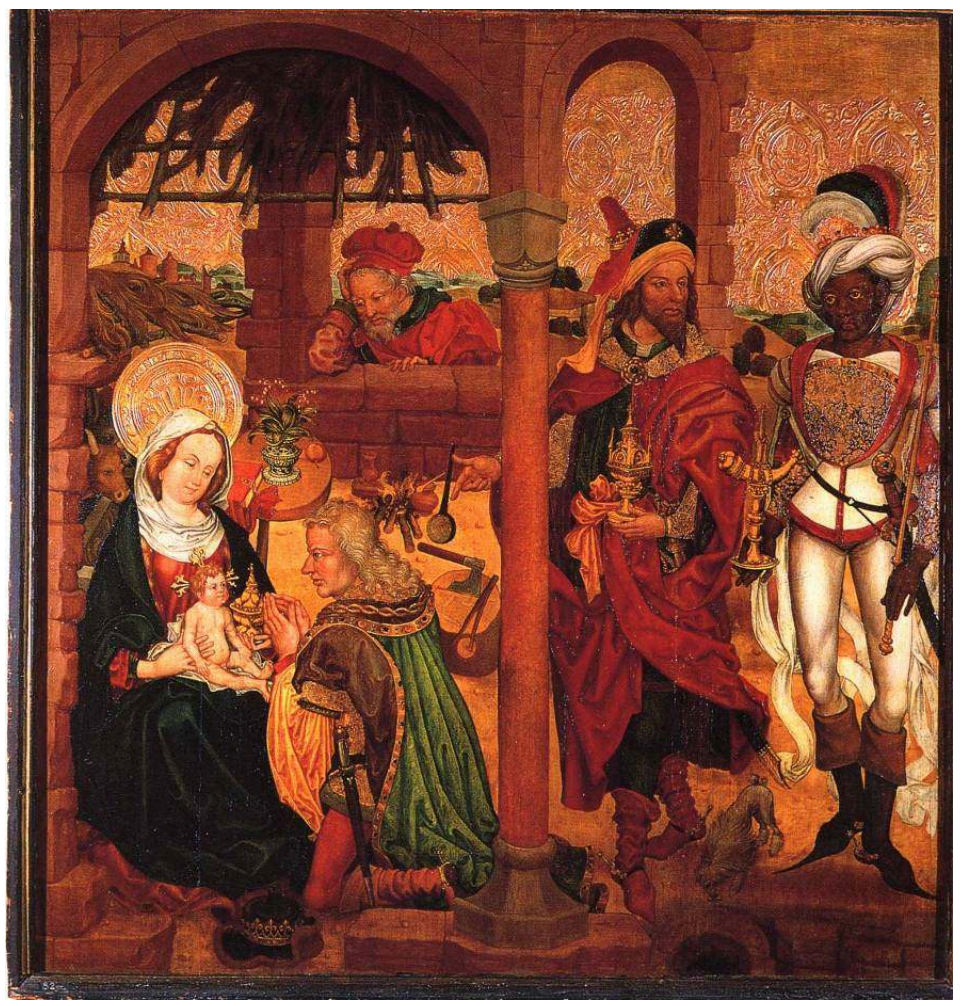

Urban Huter, Adoration des mages, 1510, Colmar, Musée de Underlinden

Dans une série de peintures, surtout nordiques, un Joseph fatigué et mélancolique est situé entre les ruines du monde antique et les promesses de celui à venir. Parfois (fig. 16), il se penche au dessus du muret qui l'exclut de l'espace où la divinité de l'enfant est reconnue et adorée par les rois mages, d'autres fois il s'occupe de la subsistance matérielle de la famille, comme quand on le voit au loin aller prendre de l'eau, (fig. 17) réparer les chaussures ou cuisiner, alors même que l'âne et le bœuf sont en adoration devant le divin enfant. 


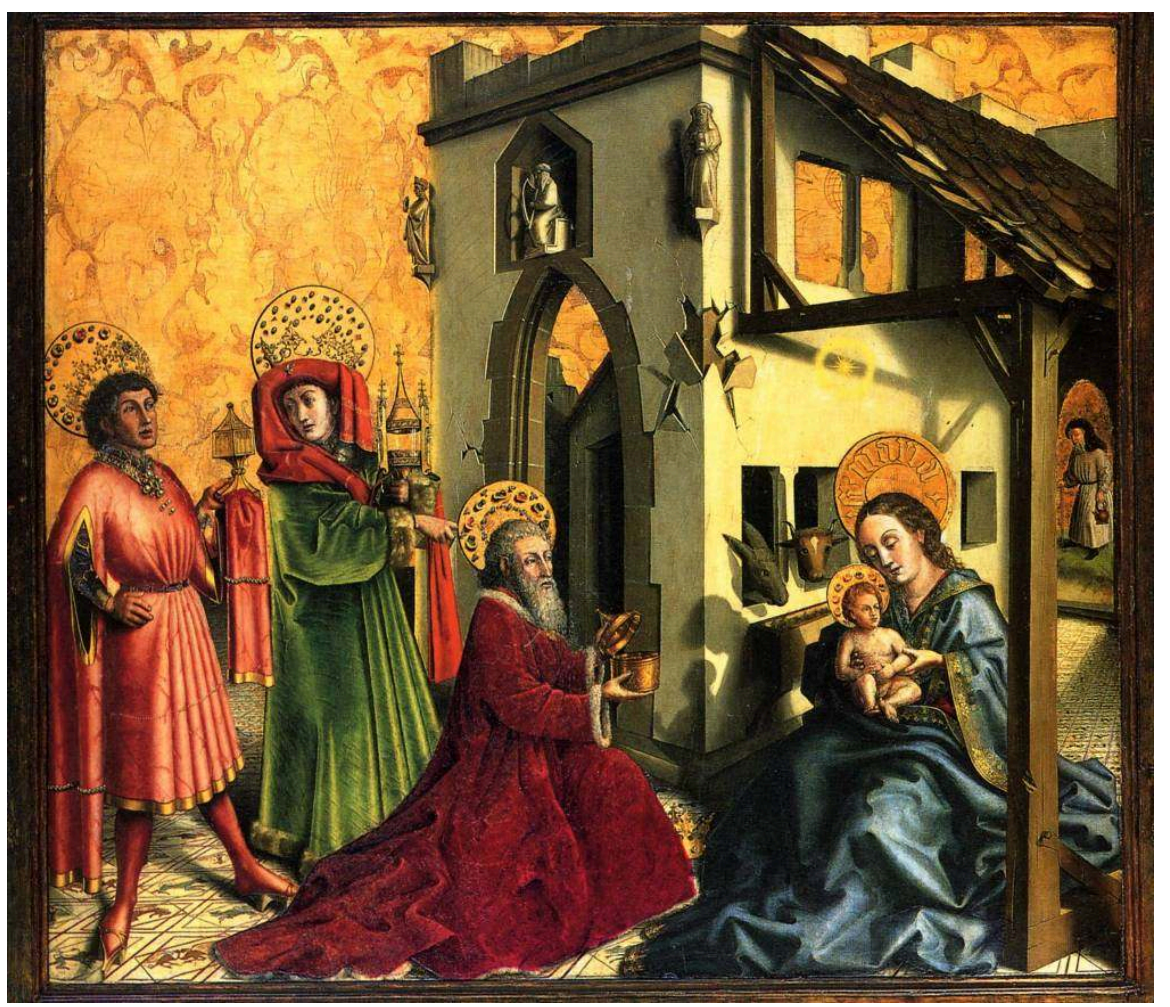

Conrad Witz, Adoration des Mages, 1444, Genève, Musée d'Art et d'Histoire

41 Il s'agit d'un motif présent dans des gravures très connues, comme celle de Dürer de 1501 qui montre Joseph alors qu'il tire l'eau du puit. En Italie, les Nativités et les Fuites en Egypte montrent le père adoptif de Jésus autrement: les traits juifs sont atténués et souvent seulement visibles à travers la couleur jaune du manteau, alors que sa vieillesse et sa torpeur mélancolique sont toujours soulignées. Dans une Nativité nocturne peinte par Gentile da Fabriano sur une prédelle aujourd'hui aux Offices (fig. 18) on voit sur la droite un Joseph profondément endormi alors que Marie veille l'enfant avec le bœuf et l'âne. A droite, deux jeunes filles sont représentées: l'une tourne le dos à la scène principale et semble, elle aussi, dormir, alors que l'autre veille et regarde l'enfant. Ces deux figures sont évidement associées aux deux personnages principaux, la jeune fille qui veille est, comme Marie, consciente du sens spirituel de cette naissance tandis que celle qui, comme Joseph, dort, incarne l'alternative « juive » ou charnelle dans la mesure où elle ne saisit pas la divinité de l'enfant et cède au désir de se reposer. 
Fig. 18

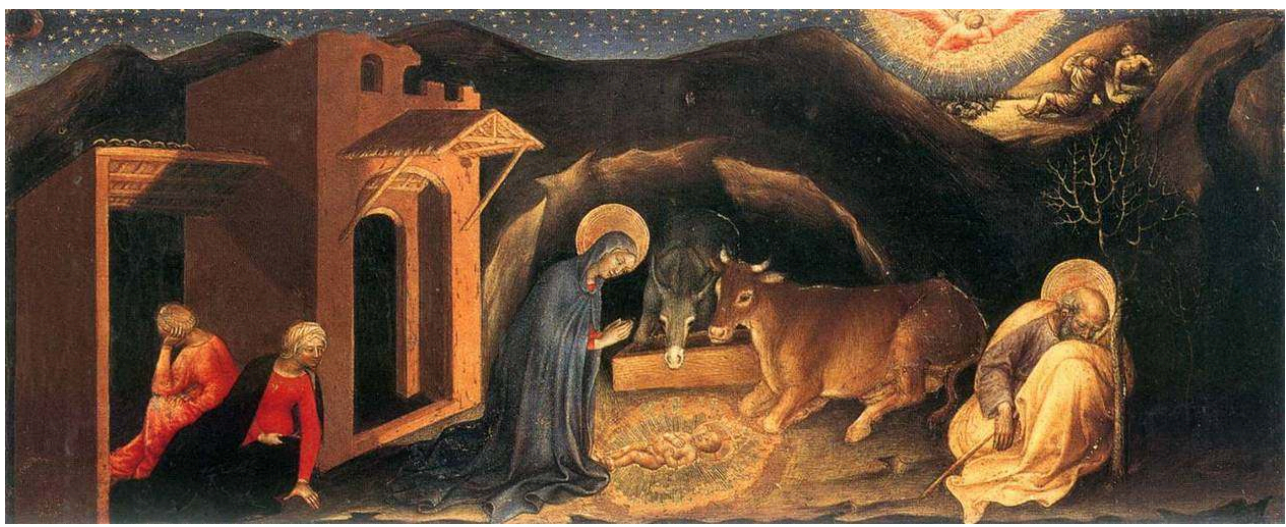

Gentile da Fabriano, Natività, 1423, Florence, Galerie des Offices

Dans la partie centrale de cette même prédelle est peinte une Fuite en Egypte où l'on retrouve les deux mêmes jeunes filles : la première porte un panier et semble vouloir aider la famille en fuite, tandis que la seconde cache derrière son dos une pomme qu'elle veut sans aucun doute garder pour elle. Leur dispute gestuelle (fig. 19) peut encore une fois être interprétée comme une opposition entre la vie secundum carnem et celle secundum spiritum; en d'autres termes, les images " pensent ", de façon autonome, le statut théologique de la "chair» dans le cadre de la rupture généalogique qui marque le passage entre l'Ancienne et la Nouvelle Ere.

Fig. 19

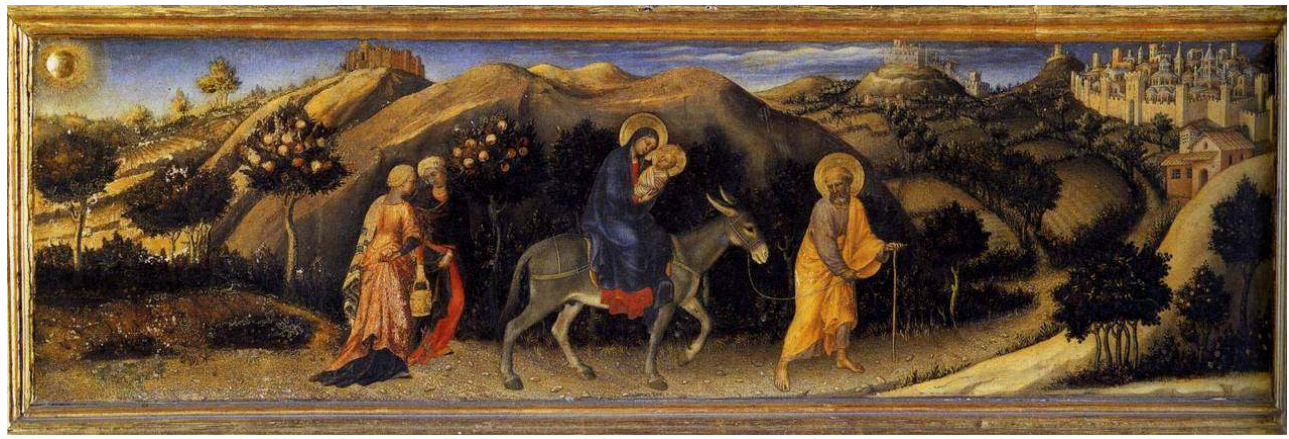

Gentile da Fabriano, Natività, 1423, Florence, Galerie des Offices

Charnel, je le rappelle, est le terme utilisé par saint Paul dans son commentaire d'un passage de la Genèse $(25,2)$ pour opposer la parenté de sang à la parenté spirituelle ou de la «promesse». Il s'agit de l'épisode dans lequel Jacob, fils de Sarah, obtient le droit d'aînesse aux dépenses de son frère Esaü, fils d'Agar. Comme la grossesse de Sarah, devenue mère à un âge très avancé suite à une intervention divine, celle de Marie est une grossesse miraculeuse et "promise» par un ange. Les juifs, écrit Paul, appartiennent à la lignée d'Esaü, par la chair (kata sarks) et seront soumis aux chrétiens comme Esaü, le frère aîné, a été soumis à Isaac, frère puîné mais préféré parce qu'il est le fils promis ${ }^{36}$.

Lorsqu'elle est utilisée avec la préposition kata, la sarks définit donc chez saint Paul une forme de vie opposée à celle qui est habitée par la présence de l'esprit, seule capable d'accueillir la promesse salvatrice du Christ. Une telle expression renvoie en particulier 
aux juifs non convertis : ceux-ci vivent secundum carnem en premier lieu parce que, ne reconnaissant pas le Messie, ils se rendent indisponibles au don de la grâce ; puis parce qu'ils observent les pratiques rituelles et les sacrifices de l'Ancienne Alliance charnellement, c'est-à-dire sans en comprendre le sens spirituel, et enfin parce qu'ils interprètent le texte sacré à la lettre et non selon l'interprétation typologique chrétienne qui y voit l'annonce de la Nouvelle Alliance. Dans un sens plus proche du nôtre, sont également charnels dans le texte de Paul, comme dans l'exégèse de saint Augustin, la sensualité et la concupiscence, vices volontiers attribués aux juifs mais qu'on reconnaît aussi comme largement diffus chez les chrétiens. Dans le contexte polémique anti-hébraïque des écrits de Paul, comme dans celui profondément différent d'Augustin, puis dans celui encore plus lointain de l'humanisme italien, la carnalité n'est pas seulement un trait juif mais, plus subtilement, une catégorie qui peut servir à décrire les mauvais chrétiens lorsqu'ils se comportent comme des juifs, assumant le trait de carnalité qui les distingue. Ainsi, par exemple, Augustin explique dans la lettre à Donatien « [q]ui sont les chrétiens semblables aux juifs » : il s'agit de ceux qui «bien que se déclarant chrétiens, sont tellement contraires à la grâce du Christ qu'ils pensent pouvoir accomplir les commandements de Dieu avec leur seule force humaine ${ }^{37}$ ». En cohérence avec l'usage de la catégorie de la carnalitéjuive à des fins de polémiques intra-chrétiennes, Erasme de Rotterdam décrit comme «juives» les pratiques extérieures et les cérémonies, en leur opposant l'intériorité du vrai chrétien ${ }^{38}$.

Il est intéressant de constater que, malgré une notable atténuation des traits négatifs et ridicules dominants au nord de l'Europe, la position marginale de Joseph est également un élément bien présent à Florence dans les années de formation de Michel-Ange. Ainsi dans une Adoration des mages de Sandro Botticelli (ca. 1470, fig. 20), le père adoptif est isolé de la foule des présents, faisant corps chromatiquement et formellement avec les ruines sur lesquelles il s'appuie. Ces restes sont les marques de la fin de l'Ere antique, sa vieillesse fatiguée porte le poids d'un âge révolu, mais elle signale aussi l'écartement de celui qui a du mal à abandonner le temps de la Loi pour entrer dans celui de la Grâce. 


\section{Fig. 20}

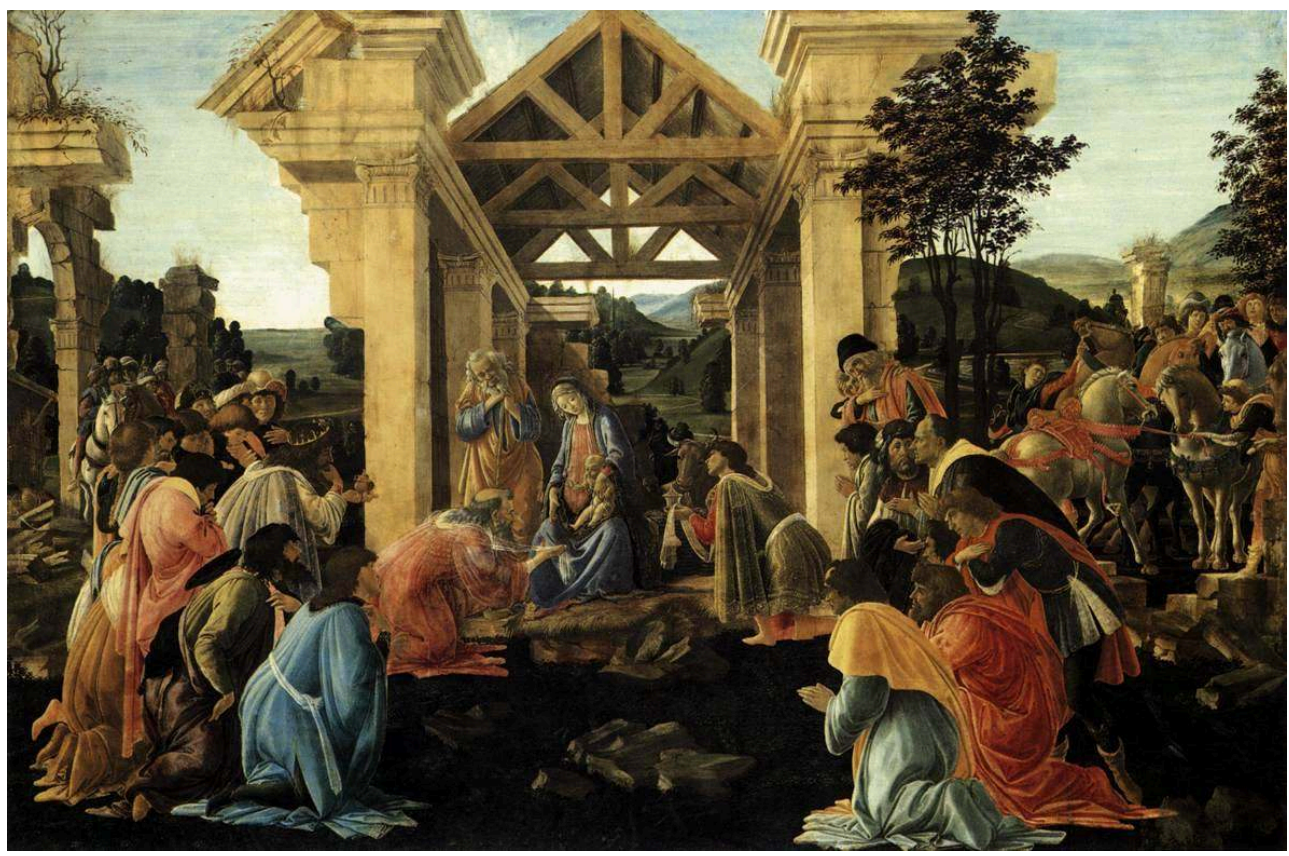

Sandro Botticelli, Adoration des mages, c. 1470, National Gallery of Art, Washington

Dans l'Evangile de Matthieu ce moment prend la forme d'un «dilemme »:

Marie, future épouse de Joseph se retrouva enceinte par l'œuvre du Saint Esprit avant qu'ils ne vivent ensemble. Son mari Joseph, qui était un homme juste et qui ne voulait pas l'accuser publiquement, pensa la répudier en secret. Alors qu'il y réfléchissait, un ange du Seigneur lui apparut en rêve et lui dit: "Joseph, fils de David, ne crains pas de prendre avec toi Marie, ton épouse. En effet l'enfant qu'elle porte est l'œuvre du Saint Esprit, elle donnera naissance à un fils que tu appelleras Jésus : il sauvera son peuple de ses péchés." 


\section{Fig. 21}

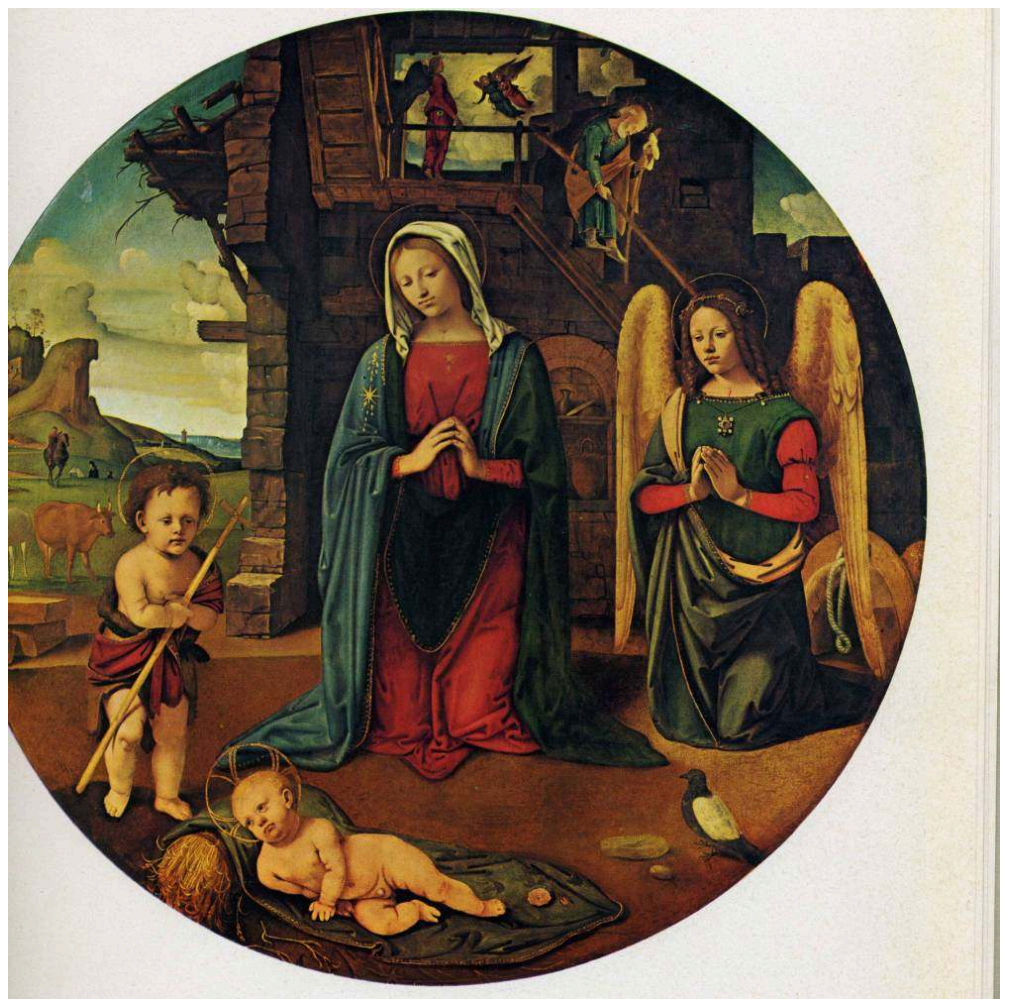

Piero di Cosimo, Adoration de l'Enfant, vers 1506, National Gallery of Art, Washington

Dans une Adoration de l'Enfant peinte vers 1506 (fig. 21), quelques années avant les fresques de la Chapelle Sixtine, Piero di Cosimo montre dans le fond un haut édifice en ruines dans lequel Joseph a à peine reçu la vision de l'Ange qui lui a communiqué la paternité divine de l'enfant. Le vieillard est exclu de l'adoration de l'enfant qui réunit toutes les figures situées au premier plan dans le tondo. Pour pouvoir rejoindre la Vierge, il doit descendre les escaliers en s'appuyant lourdement à la rampe. Le tableau souligne la lenteur de Joseph en mettant en évidence le poids de son corps alors qu'il cherche la marche de son pied gauche encore suspendu dans le vide. En retardant la participation à l'adoration du Fils, la difficile descente de l'escalier donne à voir la difficulté de passer du temps de la Loi à celui de la Grâce. La vieillesse de Joseph n'est pas seulement une caractéristique destinée à le soustraire à l'interprétation populaire qui souvent voyait en lui un mari trahi ou impuissant, mais aussi le signe de son appartenance à une époque «vieillie » et terminée. Dans le tableau de Piero di Cosimo, le lent cheminement vers Marie se charge des valeurs d'inertie et de lenteur que les chrétiens attribuaient aux juifs - et quelquefois aussi à eux-mêmes - pour leur tiédeur face à l'appel de la foi ${ }^{39}$. Je peux alors avancer deux hypothèses complémentaires : la première est que la vieillesse et la torpeur de Joseph constituent la matrice figurative de la vieillesse et de la torpeur des Ancêtres de la Chapelle Sixtine en tant que signe d'appartenance à une condition charnelle. La seconde est que les travaux domestiques effectués par le père adoptif de Jésus dans les tableaux et les gravures que nous avons examinés qualifient comme également charnels les travaux nécessaires à la subsistance décrits dans les lunettes et les cintres de Michel-Ange. 
Fig. 22

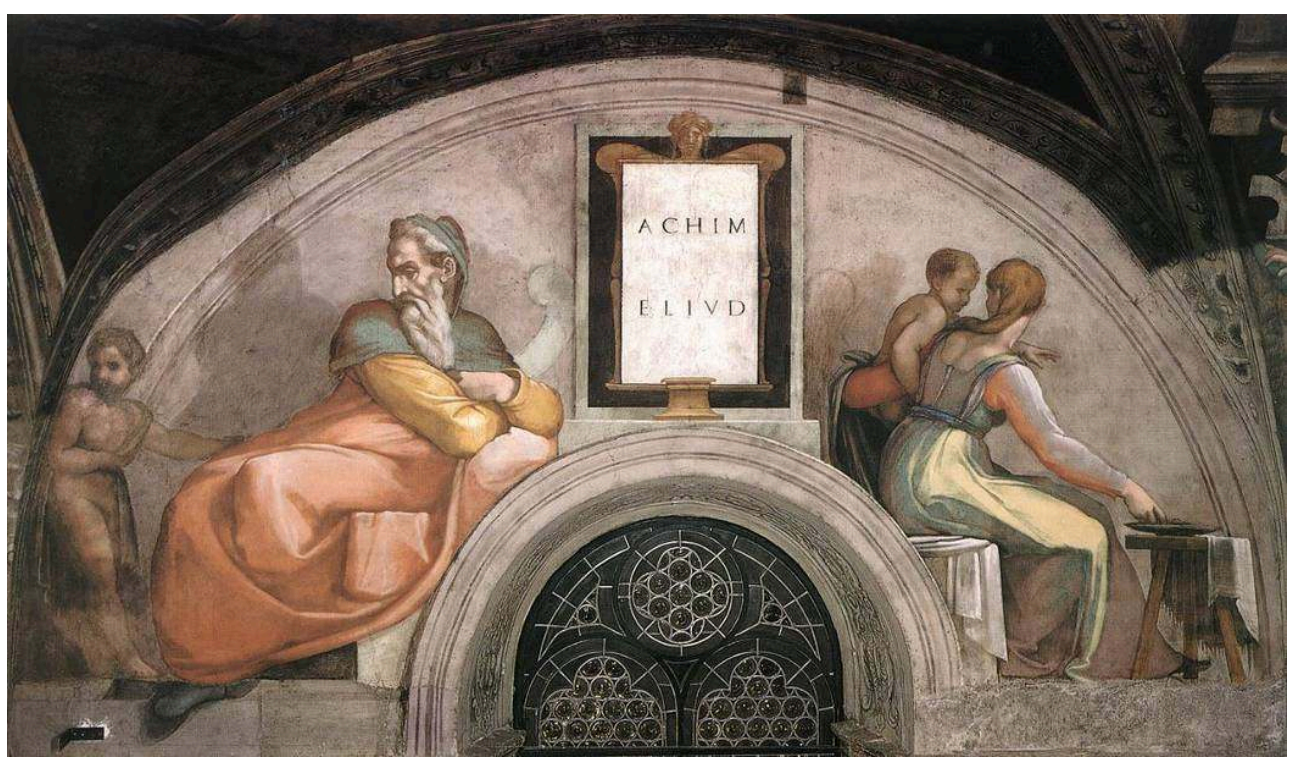

Michel-Ange, Ancêtres du Christ, 1508-12, Chapelle Sixtine, Rome

Précisons tout d'abord que les tableaux et les gravures que nous avons évoqués pour illustrer ces deux hypothèses ne sont pas les sources des ancêtres de Michel-Ange, mais qu'ils sont plutôt construits sur la base d'une structure formelle comparable et d'une opposition de valeurs similaires: d'un côté il y a ceux qui reconnaissent la venue de Dieu sur terre et saisissent le sens spirituel de chaque acte de la mère et de l'enfant, de l'autre ceux qui doutent ou hésitent à reconnaître la divinité de Jésus et, s'en tenant à une interprétation littérale des événements, s'attachent à leur aspect matériel. A partir de cette construction structurale nous pouvons déduire que les travaux et les activités domestiques nécessaire à la subsistance dans le cycle des Ancêtres de Michel-Ange n'ont pas une valeur neutre mais bien au contraire une valeur négative de distraction par rapport à la révélation de la divinité du Christ qui se manifeste partout dans les fresques de la Chapelle Sixtine. 
Fig. 23

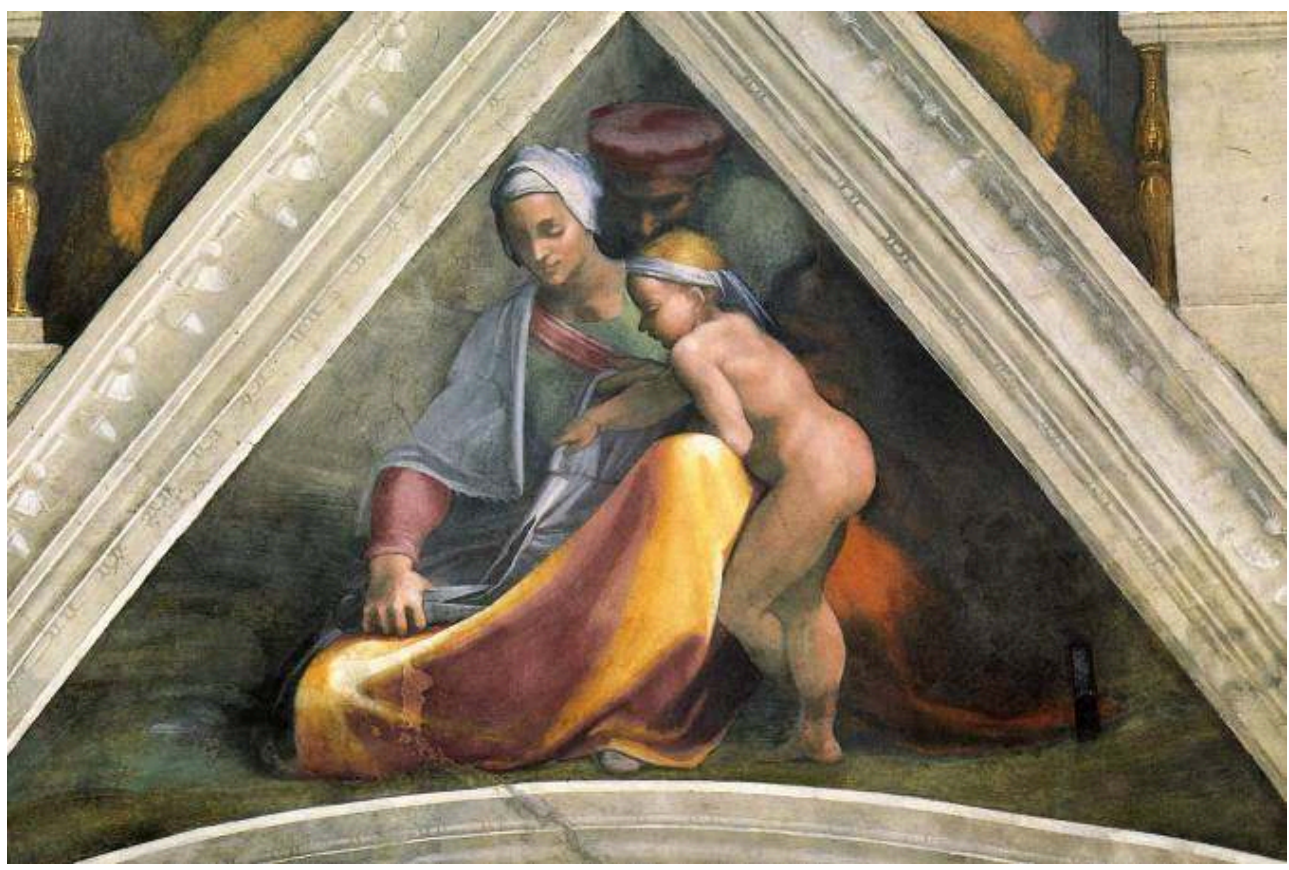

Michel-Ange, Ancêtres du Christ, 1508-12, Chapelle Sixtine, Rome

Outre l'allaitement et la préparation de nourriture, ces activités comprennent la coupe d'un vêtement et le filage. Pour le personnage féminin qui coupe un tissu sous le regard attentif de la fille et du vieux mari il est difficile de trouver des précédents iconographiques précis et convaincants. S'il s'agissait d'un geste de couture, on pourrait remonter à Marie qui, dans de nombreuses représentations, coud le voile du temple et aussi, plus rarement, un vêtement pour son fils, mais le travail de coupe de l'Ancêtre de la Chapelle Sixtine pourrait être interprété comme une opération de retouche ou d'adaptation d'un vêtement usagé pour une nouvelle utilisation. Cette lecture est orientée par ce que nous savons des activités de la grande majorité des femmes de la communauté juive de l'époque de Michel-Ange auxquelles il était interdit de s'occuper de la confection de nouveaux habits, alors qu'elles pouvaient réadapter des vêtements et tissus usés pour leur propre usage ou pour les vendre ${ }^{40}$. Si cette référence aux activités de chiffonnier pouvait être confirmée, on pourrait y voir l'un des deux - «caractères juifs documentaires » du cycle, c'est-à-dire, l'un des rares cas dans lequel les juifs ne représenteraient pas uniquement un "type» situé dans l'histoire «sans temps» de l'argumentation des théologiens, mais des juifs en partie « réels» en tant que porteurs de certains traits appartenant à un temps qu'on peut situer dans l'histoire. 
Fig. 24

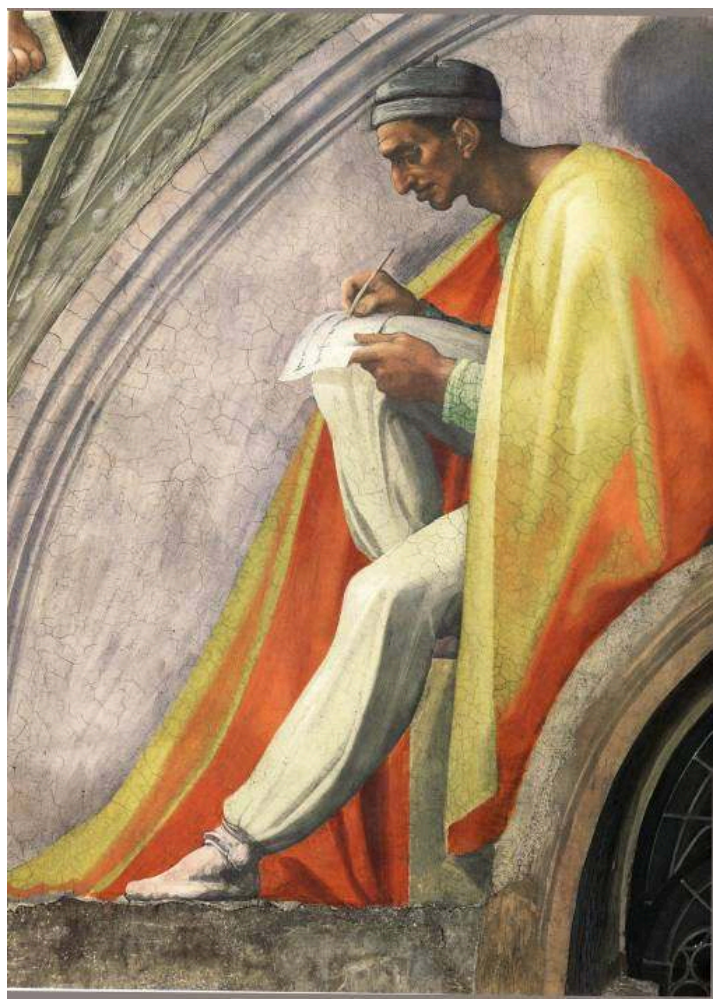

Michel-Ange, Ancêtres du Christ, 1508-12, Chapelle Sixtine, Rome

50 Le deuxième de ces traits «documentaires » pourrait être reconnu dans un usurier qu'incarne le personnage en train d'écrire dans la lunette qui porte les noms d'Asa, Iosphat et Ioram (fig. 24). Dans ce cas, le trait documentaire se charge d'une altération infamante sous forme de stéréotype avec l'allongement du cou et la déformation du nez qui renvoient aux gravures anti-juives qu'on trouve surtout dans l'Europe du nord.

51 Les activités de chiffonnier et de prêt d'argent n'avaient pas, par ailleurs, uniquement une valeur économique mais elles étaient considérées en elles-mêmes comme des travaux infamants. La première implique en effet une part d'impureté liée au contact avec les vêtements usagés et donc trop intimement associés aux corps des premiers propriétaires. La deuxième, bien que tolérée et nécessaire à l'économie, était condamnée par l'Eglise car, en tirant profit du temps écoulé entre le prêt et sa restitution, elle spéculait sur le passage du temps, lequel ne dépend pas des hommes mais de la providence divine. Le temps de l'usure est un temps soustrait au temps de l'histoire chrétienne du salut, il constitue donc, en tant que tel, une sorte d'alternative profane au modèle messianique de l'histoire chrétienne ${ }^{41}$. En d'autres termes, nous ne voyons pas seulement ici exposé à la désapprobation l'odieux juif usurier, mais plus subtilement stigmatisée, par la position de sa figure dans la chapelle Sixtine, une «façon d'être dans le temps » qui est charnelle puisqu'elle s'exclut de l'historicité chrétienne.

A travers l'analyse de la position de Joseph dans l'iconographie chrétienne nous avons commencé à mieux comprendre certains aspects des familles des Ancêtres. L'étude de ces fresques est loin d'être épuisée ${ }^{42}$. Il m'importe de souligner en conclusion le lien entre l'étrangeté de ces figures et la question de la parenté : seule la mise en travail du paradoxe généalogique, qui inclut les juifs dans l'histoire chrétienne tout en les 
excluant, permet de rendre compte de l'étrangeté comme production de l'altérité. Les juifs sont les ancêtres des chrétiens ; les Ancêtres de la Chapelle Sixtine sont la figure de cette parenté revendiquée et refusée. Voir la figure de l'Ancêtre comme le lieu d'une altération constitutive de l'identité implique alors la reconnaissance de l'exception chrétienne par rapport aux cultures qui valorisent l'ancestralité, fondant leur histoire sur la continuité avec les temps des anciens.

Une fois mise au jour la structure paradoxale de la généalogie du Christ, il nous reste à essayer de comprendre les déterminations historiques et théologiques contingentes impliqués dans l'aspect « extraordinaire et nouveau » des Ancêtres michelangelesques. Cette opération de contextualisation est fort complexe et dépasse largement les limites de cet article. Je me limiterai donc à avancer l'hypothèse que la dévalorisation du Juif et tant qu'Ancêtre a partie liée avec l'idée de réforme de l'Eglise préconisée par Jérôme Savonarole ${ }^{43}$, le prêcheur dominicain dont Michel-Ange avait écouté les discours passionnés et effrayants et dont, d'après Condivi, il avait gardé en mémoire le son de la "voix» tout le long de sa vie ${ }^{44}$. Seule la perspective d'une imminente refondation du corps ecclésial, fondée sur une authentique conversion de tous les chrétiens, permet en effet de comprendre qu'en tant que figures de la vie selon la chair, les Juifs en soient venus à assumer le rôle d'exprimer la force d'inertie qui oppose résistance et délai au processus de reforme. Il faut néanmoins noter que dans cette perspective le Juif peut assumer le rôle de représenter le "chrétien négligent » occupant ainsi la position de « tiède » propre à la majorité des chrétiens dans la vie réelle. La génération à laquelle appartiennent les Ancêtres michélangelesque aurait alors, parmi d'autres traits, ceux des chrétiens de "maintenant » : ceux qui, malgré la Révélation persévèrent dans leur " tiédeur ». En termes augustiniens cette condition est celle du seculum, la dernière des périodes de l'histoire humaine avant le Jugement Dernier. Ce n'est pas le dernier des paradoxes de constater que sous la figure de l'Autre se révèle, peut-être, celle du Même, à savoir une figure de soi «selon la chair » celle du Christianus sub specie judeorum. Ce retournement serait le fruit ultime de la rencontre entre les tensions contradictoires qui traversent le régime généalogique lorsqu'il s'agit des relations entre chrétiens et juifs et le travail d'altération propre aux figures " expérimentales », extraordinaires et nouvelles, des Ancêtres du Christ.

\section{NOTES}

1. Cet article est un extrait d'un livre que je suis sur le point de terminer et de publier, je remercie les rédacteurs d'Images re-vues de l'accueillir dans ce numéro. J'ai apporté au texte d'origine quelques adaptations minimales pour suggérer la suite de mon argument, sans cependant pouvoir présenter la totalité de mon analyse du cycle des Ancêtres du Christ.

2. Giorgio VASARI, La Vita di Michelangelo, nelle redazioni del 1550 e del 1568, dirigée et commentée par Paola BAROCCHI, Vol 1, Testo, Vol II commento, Milano, Napoli Ricciardi, 1962, note 426, p. 609-618.

3. Ibid., p. 609.

4. Emile zola, Les Trois villes, Rome, Paris, Fasquelle, 1954, p. 212. 
5. HOLROYD, Michael Angelo Buonarroti. By Charles Holroyd, keeper of the National Gallery of British Art, with translations of the life of the master by his scholar Ascanio Condivi, and three dialogues from Portugueses by Francisco de Hollanda, London, 1903, p. 177 et sv. cit in Barocchi, Commento, p. 610.

6. M. BERTINI, Michelangelo fino alla Sistina, Torino, 1942, p. $107 \mathrm{~s}$.

7. Ch.de Tolnay, Michelangiolo, Firenze, 1951, p. 47.

8. E. Edgar WIND, The religious symbolism of Michelangelo, The Sistine Ceiling, Oxford University Press, 2000, p. 12. "In Michelangelo's plan, a picture of a vice appears opposite each picture of a virtue, both of them signified by Hebrew names. The name Aminadab, for example, with which the series begins, is joined to the picture of a young girl with a veil across her lap, preoccupied with combing her hair, while a man, sitting motionless at her side, stares vacantly into space. Aminadab is translated as populus meus ('my people'), and these words occur in the larger passage: 'Can a maid forget her ornaments or a bride her attire? Yet my people populus meus have forgotten me days without number' (Jeremiah ii, 32). The couple represents the vices of worldliness and oblivion."

9. Frederick HARTT, "Lignum vitae in Medio Paradisi: The Stanza d'Eliodoro and the Sistine Ceiling," Art Bulletin $32 \mathrm{n}^{\circ} 2$ (June 1950) p. 205 "As the lunettes proceed back and forth across the chapel, the next ancestor is the young Aminadab, sitting upright and gazing fixedly into space, while his wife combs her long, blonde hair. Aminadab means "my people are willing (or spontaneous or voluntary)." The spontaneity of Christ's sacrifice was meant; Gregory and Hrabanus both quote from the Psalms: "I will freely sacrifice to thee; I will praise thy name 0 Lord, for it is good." Aminadab is placed below the Crucifixion of Haman the sacrifice of evil on the Cross must be voluntary. The only other Old Testament reference to Aminadab is in the Song of Songs (...): "I have not known: my soul hath disturbed me because of the chariots of Aminadab." To Gregory these chariots were the four Gospels, and the Synagogue was disturbed, because she did not know Christ's sacrifice. Gregory's interpretation explains the pose and glance of Aminadab, deeply disturbed by the revelation, of whose significance he had been ignorant. But who is the beautiful lady looking outward while she combs her golden hair? In the Song of Songs, shortly before the reference to Aminadab, we read: "Who is she that looketh forth as the morning, fair as the moon, clear as the sun, and terrible as an army with banners?" Not only was this taken as a prophecy of the Virgin (and therefore the Church), but the text appears in the liturgy for the Assumption, to which the chapel was dedicated. We shall find throughout the representations of the ancestors that as the men prefigure Christ, the women foretell Mary."

10. La recherche d'Edgar Wind sur la Chapelle Sixtine a commencé en 1935, mais ce n'est qu'en 1944 qu'il a publié son essai : "Sante Pagnini and Michelangelo. A study for the Succession of Savonarola”, Gazette des Beaux Arts, 1944, II, p. 211-246; l'élaboration du travail a été reconstruite par Elizabeth Sears dans les pages d'introduction au recueil des essais de Edgar Wind sur la Sixtine seulement en 2000 : Edgar Wind, The religious symbolism of Michelangelo, The Sistine Ceiling, Oxford University Press, 2000. La polémique entre Edgar Wind et Frederick Hartt a commencé après la publication de Frederick Hartt, "Lignum vitae in Medio Paradisi: The Stanza d'Eliodoro and the Sistine Ceiling," Art Bulletin 32 n 2 (June 1950) p. 115-145, 181-218. Edgar Wind a répondu avec, "Typology in the Sistine Ceiling: A Critical Statement", The Art Bulletin, vol. 33, n 1 (March.; 1951) p; 41-47, puis Frederick Hartt, "Pagnini, Vigerio, and the Sistine Ceiling: A Reply", The Art Bulletin, vol. 33, $\mathrm{n}^{\circ} 4$ (Dec., 1951) p. 262-273.

11. Sidney J. FREEDBERG, Paintings of the High Renaissance in Rome and Florence, Cambridge, Harvard University Press, 1961, p. 111.

12. On doit la tentative la plus récente à Heinrich W. Pfeiffer, mais il n'est pas plus convaincant que ces prédécesseurs. Heinrich W. PFEIFFER, La chapelle Sixtine révelée, Paris, Hazan, 2007. 
13. C. GILBERT, Michelangelo on and off the Sistine Chapel, New York, Georges Braziller, 1994, p. 115-149, E.G. DOTSON, “An augustinian interpretation of Michelangelo's Sistine Chapel”, The Art Bulletin New York, N.Y., 1979, v.61, n.2, p. 223-256 e n.3, 405-429.

14. F. MANCINELLI, De Stroebel A. M., Michelangelo. Le lunette e le vele della Cappella Sistina, De Luca, Milano, 1992, p. 7-9.

15. Barbara WISCH, 'Vested Interest: Redressing Jews on Michelangelo's Sistine Ceiling', Artibus et Historiae, $\mathrm{n}^{\circ}$ 48, 2003, p. 143-72.

16. A propos du signum cf. Robero BONFIL, Gli ebrei in Italia nell'epoca del Rinascimento, Sansoni, Firenze, 1991, Léon Poliakov, L'étoile jaune, Editions Grancher, 1999

17. Anna FOA fait une excellente synthèse historique de la condition des juifs en Occident, complétée par une ample bibliographie dans Ebrei d'Europa. Dalla peste nera all'emancipazione, Roma-Bari, Laterza, 2004.

18. A propos de la notion de "juif herméneutique", voir Jeremy COHEN, Living Letters of the Law. Ideas of the Jew in Medieval Christianity, University of California Press, 1999, Paula FREDRIKSEN, Excaecati Occulta iustitia Dei,: Augustine on Jews and Judaism" Journal of Early Christian Studies 3(1995) , 299-324. Franklin T. Harkins, "Nuancing Augustine's hermeneutical Jew: Allegory and actual Jews in the Bishop's sermons", Journal for the study of Judaism in the Persian, Hellenistic and Roman period A. 2005, vol. 36, $\mathrm{n}^{\circ}$ 1, pp. 41-64.

19. Cit. in Erich AUERBACH, Studi su Dante, Feltrinelli, Milano, 2005, p. 171.

20. Ibidem, p. 172.

21. Ibidem. p. 169.

22. Condivi écrit : «Il a particulièrement admiré Dante, charmé par l'extraordinaire génie de cet homme, qu'il avait presque entièrement à l'esprit ", cf. A. Condivi, Vita di Michelangelo Buonarroti, Rizzoli, Milano, 1964, p. 81

23. E. STEINMAN, Die Sixtinische Kapelle, II, Band: Michelangelo, München, 1905.

24. Cf. Andrea PAPPAS, "Observations on the ancestor cycle of the Sistine ceiling", Source, vol. 11, winter 1992, p. 27-31. Dans cet intéressant article, l'une des raisons de cohérence entre les cycles $\mathrm{du} \mathrm{XV}$ et les ancêtres se trouve dans la réaffirmation, dans les deux doctrines, du primat du pape contesté aussi bien sous Sisto IV que sous Jules II.

25. L.D. ETTLINGER, The Sistine Chapel before Michelangelo. Religious Imagery and Papal Primacy, Oxford, Oxford University Press, 1965. Le lien entre les Ancêtres et les Papes est confirmé dans une des lunettes détruites pour faire place au Jugement où l'un des ancêtres indiquait justement l'emblême de Jules II, signalant ainsi la continuité entre la généalogie qui précède la venue du Christ et le Pape commanditaire de l'oeuvre

26. Lisa Pona éclaircit la valeur idéologique "actuelle" de la connexion entre les papes et les Ancêtres sous les pontificats de Sisto IV et de Jules II. La référence à un passage extrait de De sacro altaris mysterio d'Innocent III (1198-1296) est particulièrement intéressante ; il y fait référence à une cérémonie d'entrée dans la chapelle papale pendant laquelle le pape et sa cour "generationis Christi seriem representat, quam Matthaeus evangelista describit". Malgré la grande distance temporelle entre cet acte liturgique et l'époque de Jules II, de nombreuses sources concordent pour indiquer la permanence des rituels dans le texte d'Innocenzo. Cf. Lisa PON, "A note on the Ancestors of Christ in the Sistine Chapel", Journal of the Warburg and Courtauld Institutes, vol. 61, 1998, pp. 254-258., la référence citée se trouve page 258, note 18.

27. D. REDIG DE CAMPOS, Itinerario pittorico dei Musei vaticani, Roma, 1954.

28. La critique de cette dimension automatique et irréfléchie de la fonction de nomination liée au portait inaugure, selon Louis Marin, la philosophie moderne du langage des Messieurs de Port Royal, cf. Louis MARIN, La Critique du discours : études sur la Logique de Port Royal et les Pensées de Pascal, Paris, Éditions de Minuit, 1975.

29. Daniel ARASSE, On n'y voit rien. Descriptions, Paris, Denoël 2000. 
30. "Resentful of infringement of his prerogative", Edgar wIND, The Religious Symbolism, cit p. 102. Pour rendre justice à Edgar Wind, il faut dire que ce travail de déchiffrage n'est pas une fin en soi mais fonctionnel à son interprétation allégorique globale de la Chapelle Sixtine.

31. Lisa PON, 'A note on the Ancestors', op. cit. p. 256.

32. SAINT AUGUSTIN, Sermon sur la montagne, Harmonies des Evangiles, $6^{\text {ème }}$ partie.

33. Karin FRIIS PLUM, 'Genealogy as Theology', Scandinavian Journal of the Old Testament, vol. 1, 1989, 90.

34. See L. D. ETTLINGER, The Sistine Chapel before Michelangelo: Religious Imagery and Papal Primacy, Oxford, 1965 ; Rona Goffen, 'Friar Sixtus IV and the Sistine Chapel', Renaissance Quarterly, 39 : 2, 218-262, Deoclecio Redig de Campos, 'I Tituli' degli affreschi del Quattrocento nella Cappella Sistina', Rendiconti della Pontificia Accademia Romana de Archeologia, vol. 42, 1969-70, 229-31; see also Gerhard RUF, S. Francesco e S. Bonaventura, Assisi, 1974. Voir aussi Armando PETRUCCI, La scrittura : ideologia e rappresentazione, Torino, Einaudi 1986.

35. Ruth MELLINKoff, Outcasts: Signs of Otherness in Nourthern European Art of Late Middle Ages, University of California Press, 1993.

36. Cf. Carlo GINZBURG, "Un lapsus di papa Woytila", in Occhiacci di legno.Nove riflessioni sulla distanza, Milano, Feltrinelli,1998, p. 210-216.

37. SAINT AUGUSTIN, Lettre à Donatien, (lettre 196)

38. Simon MARKISH, Erasme et les juifs, Paris, L'Age d'Homme, 1979

39. Un célèbre exemple de tiédeur et de doute se trouve dans le Secretus de Pétrarque.

40. Cf. Leon Poliakov, Histoire de l'antisémitisme, t. I : Du Christ aux Juifs de Cour, Paris, CalmannLévy, 1955.

41. Ibidem.

42. Il me faut interrompre ici une argumentation qui ne peut être, pour des raisons d'espace et de pertinence, entièrement développée. Mon travail propose en particulier une analyse comparée de la position des Ancêtres dans la Chapelle Sixtine et de la position de la communauté juive romaine face au Pape. Cette réflexion dérive d'une analyse formelle des "rencontres rituelles" entre le Pape et les juifs. Entre autres, j'aborde la question de la mélancolie des Ancêtres à travers une enquête sur la contamination de l'iconographie de Saturne et de celle du juif. De plus, je me penche sur la question de l'iconographie de la famille et de la maternité en particulier, mais également sur la figure du pèlerinage. La conclusion de l'analyse revient enfin sur la "négligence" et sur "l'inertie" des Ancêtres du Christ à travers une archéologie de l'inertie spirituelle qui conduit à l'hypothèse selon laquelle il est possible de trouver dans la fresque un autoportrait de Michel-Ange sous les traits d'un juif dont il partage une forme d'inertie spirituelle. Cette lecture autopunitive est confirmée par une étude sur certaines des dernières poésies de l'artiste et relance une réflexion sur la figure du chrétien 'charnel' sub specie iudeorum.

43. Voir à ce propos: Kenneth stow, 'The Papacy and the Jews: Catholic reformation and Beyond', Jewish History ${ }_{2}$ Vol 6 n 2, 1992, p. 259-260 «Le $\mathrm{XVI}^{\mathrm{e}}$ siècle traverse un processus de restructuration et de redéfinition conscientes. A la différence de l'Eglise médiévale qui se concevait comme coextensive à la société médiévale, l'Eglise qui émerge à la fin du $\mathrm{XvI}^{\mathrm{e}}$ siècle se présente comme un corps simplifié et moins prétentieux. Cette dimension réduite était avantageuse. Au Moyen Age, dans l'extension qui était alors la sienne, l'Eglise avait été forcée d'exister dans la diversité. Dans la nouvelle identité qu'elle a acquise au $\mathrm{xvI}^{\mathrm{e}}$ siècle, elle peut se montrer plus rigide et s'atteler à la transformation en une réalité effective d'une idée de Réforme jusque là éludée, prônant une "Eglise pure et unifiée ", conduite par un clergé également pur, dans une société complètement catholique. L'idée d'une telle Eglise, dont l'accomplissement était une ambition cléricale depuis l'époque de saint Benoît et de saint Augustin mais que l'ampleur de l'Eglise médiévale ne pouvait mener à son terme, entre dans un processus de réalisation dès la 
deuxième décade $\mathrm{du} \mathrm{xvI}^{\mathrm{e}}$ siècle. Dans ce cadre, la place de juifs qui avaient toujours été des symboles de l'impureté et de la division de l'Eglise devait être reconsidérée ", je traduis.

44. Ascanio CoNDIVI, Vita di Michelagelo Buonarroti,(1553) Florence, 1998 (chap. LVI)

\section{RÉSUMÉS}

Parmi les milliers de personnages peints dans la Chapelle Sixtine, ceux qui sont représentés dans les lunettes et les cintres de la voûte semblent être sans rapport ni de temps ni de lieu avec le contexte héroïque de l'ensemble. On y voit des familles prises dans leur vie intime, des femmes s'occupant de leurs enfants ou de travaux domestiques, des vieillards mélancoliques endormis, des personnages errants, des hommes et des femmes dans l'attente. Nombre d'entre eux ont les bras et les jambes croisés, comme pour témoigner d'un empêchement à l'action. Leurs figures accablées projettent sur les murs des ombres denses, autant de marques d'une corporéité terrestre et d'une « immersion » dans le temps de la vie de tous les jours. Certaines de ces figures ont des traits sémites stéréotypés, marqués au point de rappeler certaines représentations antihébraïques de l'époque. Si l'on se situe dans une perspective d'anthropologie de la parenté et que l'on s'interroge sur les critères qui président au passage du nom et du pouvoir entre père et fils on constate que l'intervention de Dieu dans l'Incarnation et la naissance de Jésus provoquent une rupture, qui annule définitivement la valeur de la transmission par le sang propre à la généalogie charnelle, ainsi que la façon dont elle organise le cours de l'histoire. Ce n'est pas un hasard si la liste des ancêtres du Christ se trouve, au début du livre de Mathieu, précisément à l'endroit où l'auteur doit gérer l'articulation entre l'époque de l'Ancien Testament et celle du Nouveau. Dans cet article j'étudie le lien entre l'étrangeté des Ancêtres situés aux marges de la voûte de la Chapelle Sixtine et la question de la parenté : seule la mise en travail du paradoxe généalogique, qui inclut les juifs dans l'histoire chrétienne tout en les excluant, permet de rendre compte de l'étrangeté comme production de l'alterité. Les juifs sont les ancêtres des chrétiens ; les Ancêtres de la Chapelle Sixtine sont la figure de cette parenté revendiquée et refusée. Voir la figure de l'Ancêtre comme le lieu d'une altération constitutive de l'identité implique alors la reconnaissance de l'exception chrétienne par rapport aux cultures qui valorisent l'ancestralité, fondant leur histoire sur la continuité avec les temps des anciens alors que l'Incarnation introduit, pour les chrétiens une discontinuité fondatrice.

Among thousands of characters depicted in the Sistine Chapel, those of the ancestors of Christ, seem to have no relationship with the heroic context of the whole painting. One can view intimate scenes of family life, with women, children, old men. Many of them have their arms and legs crossed, as if prevented to move. Some of them have stereotyped Semitic features which recall anti-hebraic depictions of that time. As far as anthropology of kinship is concerned, the Incarnation creates a rupture: it cancels the value given to the transmission by the blood in father-son relationships. It is not by chance that, in the Gospel by Matthew, the list of the ancestors of Christ is situated at the articulation between the epochs of the Old Testament and the New. This article studies the relationships between the strangeness of the ancestors of Christ depicted in the margins of the Sistine Chapel, and the question of kinship. Only by questioning this genealogical paradox, which include the Jews in Christian History and exclude them at the same time, can one understand this strangeness as a production of alterity. 
INDEX

Keywords : ancestors, Sistine Chapel, Michelangelo, kinship

Mots-clés : ancêtre, Chapelle Sixtine, Michel-Ange, Parenté

Index chronologique : Renaissance

Index géographique : Italie

Thèmes : anthropologie des images

\section{AUTEUR}

\section{GIOVANNI CARERI}

Directeur du CEHTA, Giovanni Careri est directeur d'études à l'EHESS, membre associé au Laboratoire d'anthropologie sociale (EHESS, CNRS, Collège de France) et Professeur à l'Ecole des beaux-arts de Lyon. Il est responsable avec Bernhard Rüdiger du groupe de recherche « Art contemporain et temps de l'histoire » (CEHTA-EHESS / Ecole des beaux-arts de Lyon). Ses recherches portent toujours sur des objets singuliers et singulièrement complexes : le 'montage' des arts dans les chapelles baroques du Bernin, le réseau des tableaux, pièces de théâtre et ballet qui ont repris la Jérusalem Délivrée du Tasse et, plus récemment, la fabrique de l'histoire chrétienne dans la Chapelle Sixtine. Son approche historique et critique a recours aux outils et aux questions de l'anthropologie et de la sémiotique. 